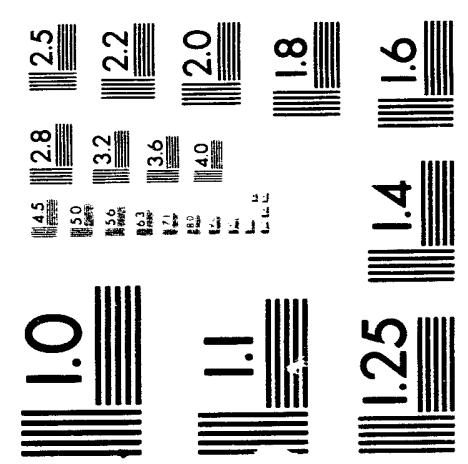



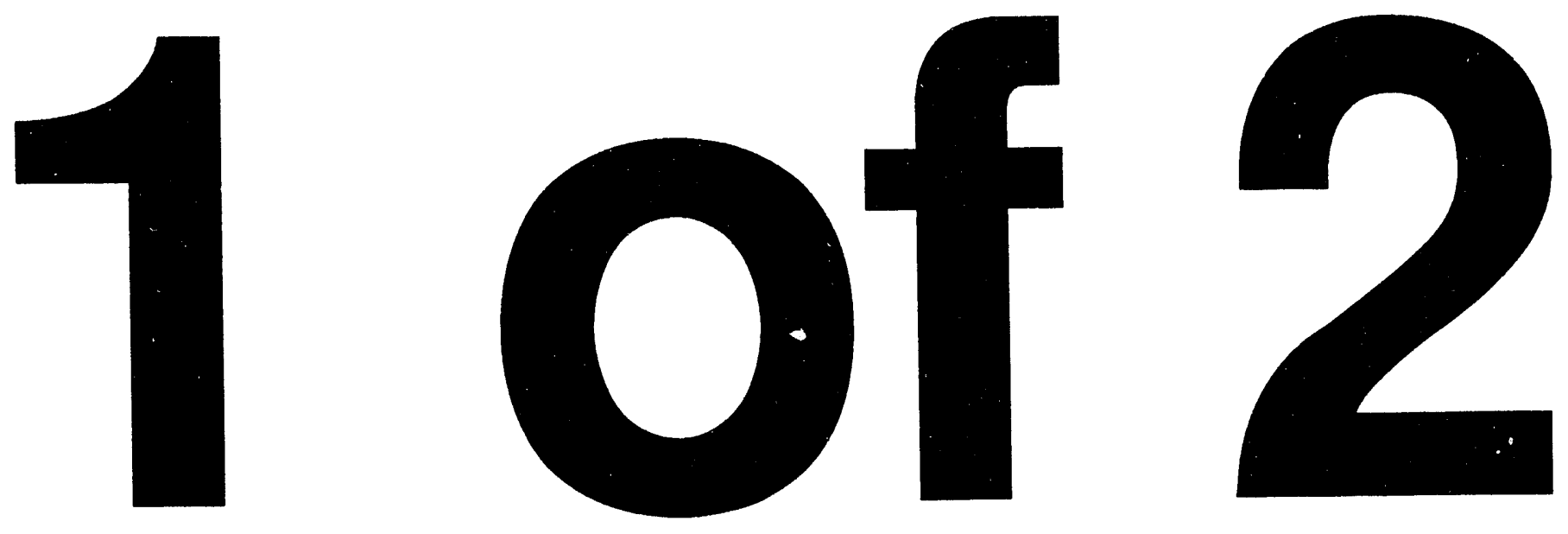


\title{
Chemically Modified Polymeric Resins for Solid-Phase Extraction and Group Separation Prior to Analysis by Iiquid or Gas Chramatography
}

by Iuther พ. Sctmidt

\author{
PhD thesis submitted to Iowa State University \\ Ames Laboratory, U. S. DOE \\ Iowa State University \\ Ames, Iowa 50011
}

Date Transmitted: July 1993

\author{
Prepared for the U. S. Department of Energy \\ Under contract no. W-7405-eng-82.
}

\section{DISCLAIMER}

This report was prepared as an account of work sponsored by an agency of the United States Government. Neither the United States Government nor any agency thereof, nor any of their employees, makes any warranty, express or implied, or assumes any legal liability or responsibility for the accuracy, completeness, or usefulness of any information, apparatus, product, or process disclosed, or represents that its use would not infringe privately owned rights. Reference herein to any specific commercial product, process, or service by trade name, trademark, manufacturer, or otherwise does not necessarily constitute or imply its endorsement, recommendation, or favoring by the United States Government or any agency thereof. The views and opinions of authors expressed herein do not necessarily state or reflect those of the United States Government or any agency thereof. 
Chemically modified polymeric resins

for solid-phase extraction and

group separation prior to analysis by

liquid or gas chromatography

Luther w. Schmidt

Under the supervision of Dr. James S. Fritz

From the Department of Chemistry

Iowa State University

Polystyrene divinylbenzene (PS/DVB) was chemically

modified by the introduction of acetyl, sulfonic acid, and quaternary ammonium groups for solid-phase extraction (SPE).

These groups increase the hydrophylicity of the resin surface, making it more efficient for the extraction of polar organic molecules from liquid samples.

A resin functionalized with an acetyl group was impregnated in a polytetrafluoroethylene membrane and used for the extraction and concentration of phenolic compounds from aqueous samples. The introduction of the acetyl group created a surface easily "wetted", making it a very efficient adsorbent for polar compounds in water. The membrane stabilized the resin bed, allowing for more efficient kinetics and thus higher flow rates and iaxger sample volumes.

Partially sulfonated high surface area resins are used 
to perform an extraction and group separation of an aqueous mixture of neutral and basic organics. The bases are adsorbed electrostatically to the sulfonic acid groups on the resin. The neutrals are adsorbed hydrophobically onto the unreacted surfaces of the resin. A two-step elution process is then used to separate the two fractions. The neutral fraction is first eluted with an organic solvent, followed by the basic fraction with a basic organic eluant. similar separations are performed in non-aqueous eluant.

A partially functionalized anion exchange resin is used to concentrate and separate organic acids and phenols from neutrals. The acids are adsorbed electrostatically to the quaternary amine groups on the resin. The neutrals are adsorbed due to hydrophobic interactions with the unreacted surfaces of the resin. The $\mathrm{pH}$ of the aqueous solution is raised sufficiently to ensure that the acids are present in their anion form, causing them to be electrostatically bound to the anion exchange groups on the resin. The neutrals are eluted first with an organic solvent, followed by the acids in an acidic organic eluant. Carboxylic acids are analyzed by HPLC and phenols are analyzed by GC. Similar separations have been performed in nonpolar media, with the neutral fraction being unretained. 


$$
\begin{aligned}
& \text { Chemically modified polymeric resins } \\
& \text { for solid-phase extraction and } \\
& \text { group separation prior to analysis by } \\
& \text { liquid or gas chromatography }
\end{aligned}
$$

by

\author{
Luther W. Schmidt
}

\author{
A Dissertation submitted to the \\ Graduate Faculty in Partial Fulfillment of the \\ Requirements for the degree of \\ DOCTOR OF PHILOSOPHY
}

Department: Chemistry
Major: Analytical Chemistry

Approved :

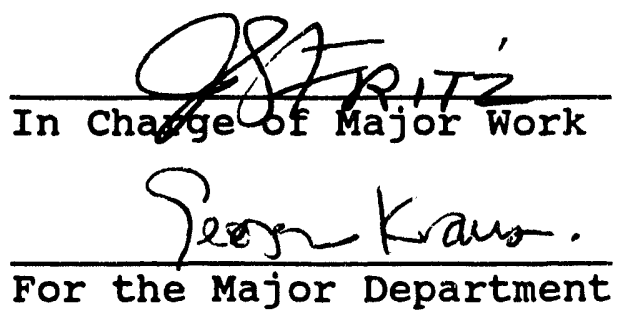

For the Graduate college

Iowa state University
Ames, Iowa

1993 
TABLE OF CONTENTS

GENERAL INTRODUCTION . . . . . . . . . . . . . 1

Polymeric Resins . . . . . . . . . . . . 1

Solid Phase Extraction . . . . . . . . . . 5

SPE with Resin Impregnated Membranes . . . . . . 22

PART I. SOLID-PHASE EXTRACTION OF PHENOLS

USING MEMBRANES LOADED WITH MODIFIED

POLYMERIC RESINS . . . . . . . 26

INTRODUCTION . . . . . . . . . . . . . 27

EXPERIMENTAL . . . . . . . . . . . . . . 32

Apparatus . . . . . . . . . . . . . 32

Reagents and Chemicals............ 34

Preparation of Modified Resins . . . . . . . . 34

Procedure for SPE . . . . . . . . . . 35

RESULTS AND DISCUSSION . . . . . . . . . . . 38

Deionized water ........... . 39

Tap and River Waters . . . . . . . . . . . 42

CONCLUSION . . . . . . . . . . . . . . 48

PART II. ION EXCHANGE PRECONCENTRATION

AND GROUP SEPARATION OF BASIC

AND NEUTRAL ORGANIC COMPOUNDS ... . 49

INTRODUCTION . . . . . . . . . . . . . 50

EXPERIMENTAL . . . . . . . . . . . . . 54

Reagents and Chemicals... . . . . . . . 54

Preparation of Cation Exchange Resin . . . . . 54 


\section{iii}

Procedure for SPE . . . . . . . . . . . 56

RESULTS AND DISCUSSION • • • • • . • • • . . • . . . 59

Group Separation of Neutral and Basic Compounds . . 59

Group Separation of Bases and Neutrals in Non-polar Solution ................ 68

Ion Exchange Separation of Organic Bases in Non-aqueous Media . . . . . . . . . . . . 75

on-line separation ..... . . . . . . . . 80

CONCLUSION

PART III. ION EXCHANGE PRECONCENTRATION AND GROUP SEPARATION OF ACIDIC AND

NEUTRAL COMPOUNDS .......... 88

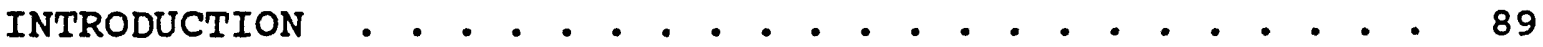

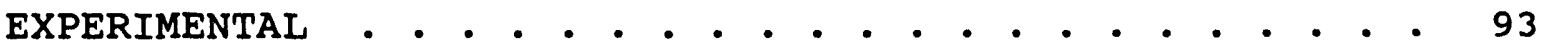

Reagents and chemicals . . . . . . . . . . 93

Procedure for SPE . . . . . . . . . . . 95

RESULTS AND DISCUSSION • • • • • • • • • • • • • • • • • 98

Group Separation of Acids and Neutrals . . . . . . 98

Group Separation of Acids and Neutrals in Non-polar

Media . . . . . . . . . . . . . 101

CONCLUSION . . . . . . . . . . . . . . 110

GENERAL CONCLUSION . . . . . . . . . . . . . . . . 111

REFERENCES • • . . . . . . . . . . . . . . . . 115

ACKNOWLEDGEMENTS • • • • . . • • • • • . • . • . • 119 


\section{CHAPTER 1. GENERAL INTRODUCTION}

This research is presented in three parts. The first section deals with the use of derivatized resins for the extraction of phenols from aqueous samples. A resin modified by the addition of an acetyl group is impregnated in a membrane and analysis of several different water samples is performed.

In Part II a cation exchange resin is used to concentrate and fractionate bases and neutrals in aqueous samples. A multi-step elution process is used to fractionate neutrals, strong and weak bases. This same resin is also used to extract bases from nonpolar solutions.

Part III deals with the use of anion exchange resin for the concentration and group separation of acids and neutrals in aqueous solution. A variety of neutrals, phenols and carboxylic acids was studied. A two-step elution process was used for group separation. This resin was also used for the extraction of carboxylic acids from nonpolar solutions.

\section{Polymeric Resins}

The most common packing used today in Solid Phase Extraction (SPE) and High Performance Liquid Chromatography (HPLC) is silica gel. The gel is a network of silica atoms bound to one another by siloxane bonds. 
This gel is then modified with any number of chemical entities such as $c 8$ or $c 18$ reacted at terminal hydroxyl groups to facilitate reverse-phase separations. Although these packings dominate the chromatography industry, as new and better polymers are being developed an increasing percentage of the market is turning to polymeric resins. Although silica columns are very efficient, they do have some serious drawbacks. The most serious of these is their instability in alkaline or highly acidic solution. They also require an extended equilibrium time between runs when gradient elution in used. These packings possess residual silanol groups which cause peak broadening and tailing of polar compounds such as amines or alcohols.

Modern polymeric resins are unaffected by $\mathrm{pH}$. In the past the big disadvantage of polymeric resins was a shrinking or swelling of the resin when subjected to solvent changes. Modern polymeric stationary phases are highly cross-linked, which gives them an increased stability towards changes in solvent strength. The most widely used polymeric resin is polystyrene divinylbenzene (PS/DVB). The chemical structure of this polymer is shown in Figure 1. Chemical derivatisation is easily achieved by Friedel-crafts reaction on the benzene ring. 
F. Nevejans et al. studied pore structure and its influence on polymeric stationary phase (7). They found that the microporosity was important to the chromatographic performance of the polystyrene. For hydrophobic solutes, the entire bulk of the resin entered into the adsorptive process, not only the surface. Solutes can penetrate the pores into the polystyrene matrix. The micropores vary with the nature of the solute and the eluent.

R. I. Greyson and A. M. Patch (8) compared PS/DVB and sic $_{18}$ for HPLC. They found that of the two reverse phase materials, the polymeric resin gave greater selectivity, higher capacity, but lower efficiency when compared to the silica resin.

A 1990 paper by Sun and Fritz (18) introduced a number of chemivally modified polymeric resins for high-performance liquid chromatography. The resin was easily derivatized by a Friedel-crafts reaction on the benzene ring of the polymer. These modifications included a hydrophilic acetyl group, a methyl cyano group, a tert-butyl group, and succinic acid group. Extensive studies were done in which the retention of various compounds was compared on the resins. The hydrophilic resins showed an increased retention of some polar compounds when compared to underivatized polymeric resins. 


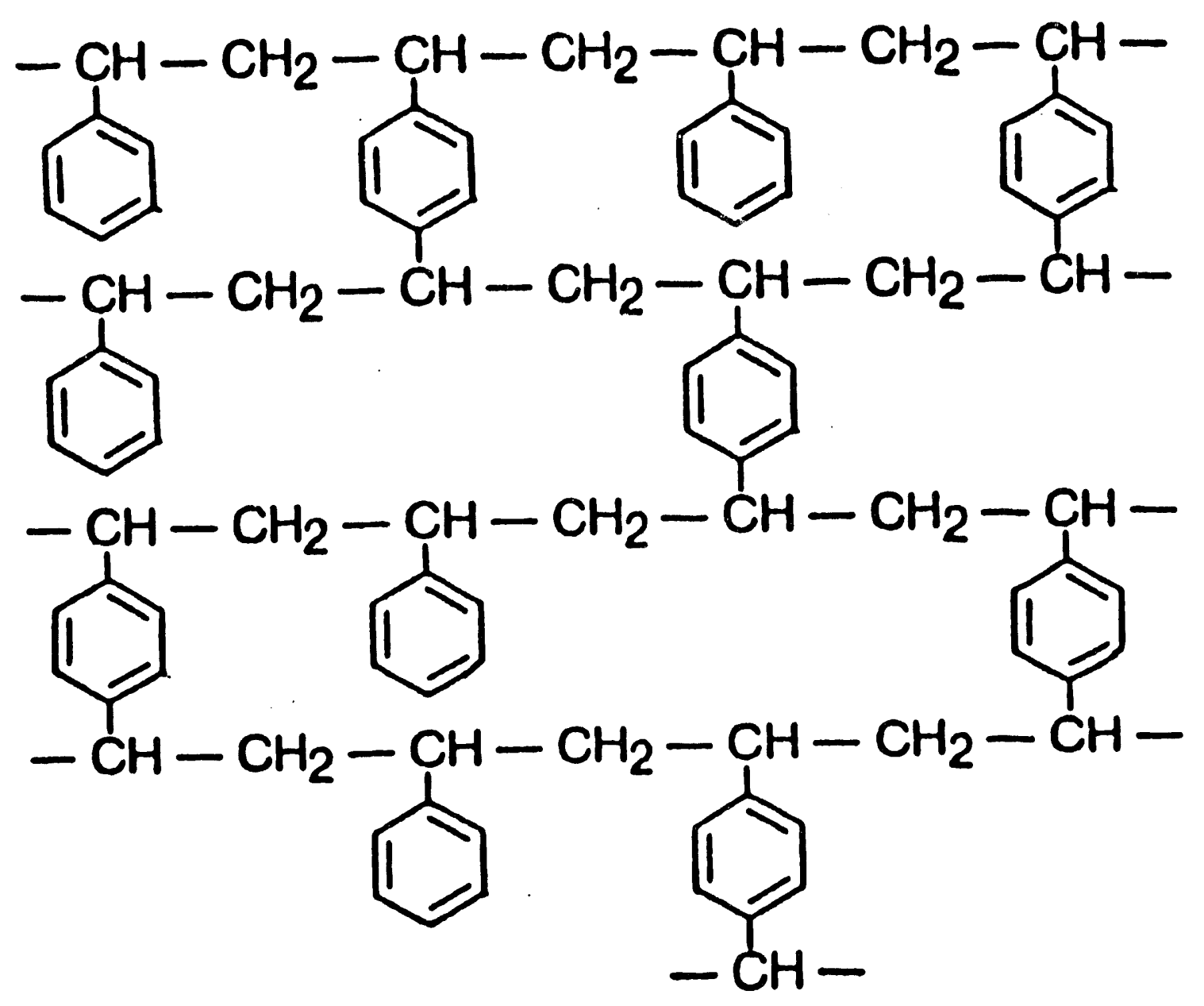

Figure 1. Chemical structure of polystyrene divinylbenzene. (PS/DVB) . 


\section{8olid Phase Extraction}

In most instances, "real world" (i.e. industrial byproduct, environmental or biological samples, etc.) samples tend to be too dilute, too complex, or incompatible with chromatographic systems. This fact is often overlooked in analytical development, thus causing sample preparation techniques to be a limiting factor in many analyses. In comparison with other developments, it has been suggested that sample preparation advances have come about due more to need rather than desire (34). Regardless of original sample concentration and matrix, the ultimate goal of any sample preparation technique is a final solution with an enriched sub-fraction of analytes of interest at easily separated and detected concentrations that are compatibile with the chromatographic system of choice.

In a recent paper on organochlorine analysis (35), D. E. Wells and an IUPAC commission pointed out the major considerations in sample cleanup, extractions, and group separations. In trace analysis the concentration of the determinand is often close to the limit of detection (L.O.D.). Qualitative analysis requires a signal three times that of the baseline noise. The limits for quantitation are generally 5-10 times greater than the L.O.D. Background interference from the sample matrix, solvent, and induced 
contamination adversely affect this signal/noise ratio. In order to minimize these influences, the general standards of good laboratory practice are essential to maintain sample integrity. Wells and his colleagues laid out the guidelines which a laboratory should follow regarding cleanliness, sample storage, and sample handling.

In the analysis of liquid samples, invariably the sample preparation step is the most time consuming step in the procedure. It is often the step in which the most analyte is lost and the majority of contaminants are introduced (via solvents, glassware, etc.). The standard E.P.A. methods for concentration and isolation of trace analytes in liquid samples call for liquid-liquid extraction. This technique is often considered to be a "mature" technology. But as pointed out in a review by Baird (9), many problems with the technique still exist. They include mass transfer of analyte in drop swarms, variation of drop size, formation of emulsions, effect of scale upon axial mixing, slow phase separation in extraction due to normally low differences in density between liquid phases and high viscosity, problems providing complete agitation of the sample to ensure intimate contact between the two phases, etc. These problems increase in importance as sample size increases. This paper illustrates the need for the development of better methods. 
7

Liska and coworkers (1) have also pointed out other problems including the problems introduced when larger solvent volumes are used. Analytical chemists today realize that the large volumes of solvents generated in today's laboratories can add to environmental problems. Not only are solvents expensive to purchase, but their disposal is costly. In addition, trace contaminants present in the extracting solvent often interfere with the measurement of the analyte of interest.

In liquid/liquid extraction concentrations are performed by boiling or evaporating off the extracting solvent. This step can lead to significant loss of analyte, not to mention the hazards involved in heating toxic and flammable solvents. Trace contaminants in the organic solvent are also concentrated, causing them to become major interferences in the final chromatographic analysis. This step can also be very time consuming.

Extraction from liquid onto a solid adsorbent is a viable alternative to liquid/liquid extraction. Fritz and Junk (5) first described the use of polymeric resins for the extraction of phenols from aqueous solutions. A small column was filled with Rohm \& Haas XAD-2 resin, and aqueous samples containing a variety of organic compounds were passed through these columns. These compounds were adsorbed onto the column 
by hydrophobic attraction and subsequently washed from the column with a small amount of organic solvent.

In contrast, solid phase extraction offers a viable alternative to standard liquid/liquid extraction. SPE offers decreased solvent consumption, greater concentration factors, increased extraction efficiency, and is more easily automated.

An example of an SPE column is shown in Figure 2. Approximately $10 \mathrm{mg}$ of resin is supported above and below by two PTFE frits. The column dimensions are $55 \mathrm{~mm}$ by $7 \mathrm{~mm}$ i.d. In Figure 3 we see a solution of the organic dye Arsenazo III passing through the resin. A tight band forms at the top of the resin as the complex is adsorbed on the resin by a hydrophobic interaction. By looking at the absence of color in the drop emerging from the column, we can see that the organic dye is completely taken up onto the resin. Figure 4 shows the elution of the dye in an acetone solution containing ammonia. By adsorbing from a large volume of aqueous solution and eluting with small volumes of organic solvents, significant concentration factors can be obtained.

As stated earlier, SPE is compatible with automated techniques. When compared to liquid/liquid extraction, which is often quite labor intensive with rather elaborate systems 
Figure 2. Photograph of SPE column containing acetyl membrane plug 


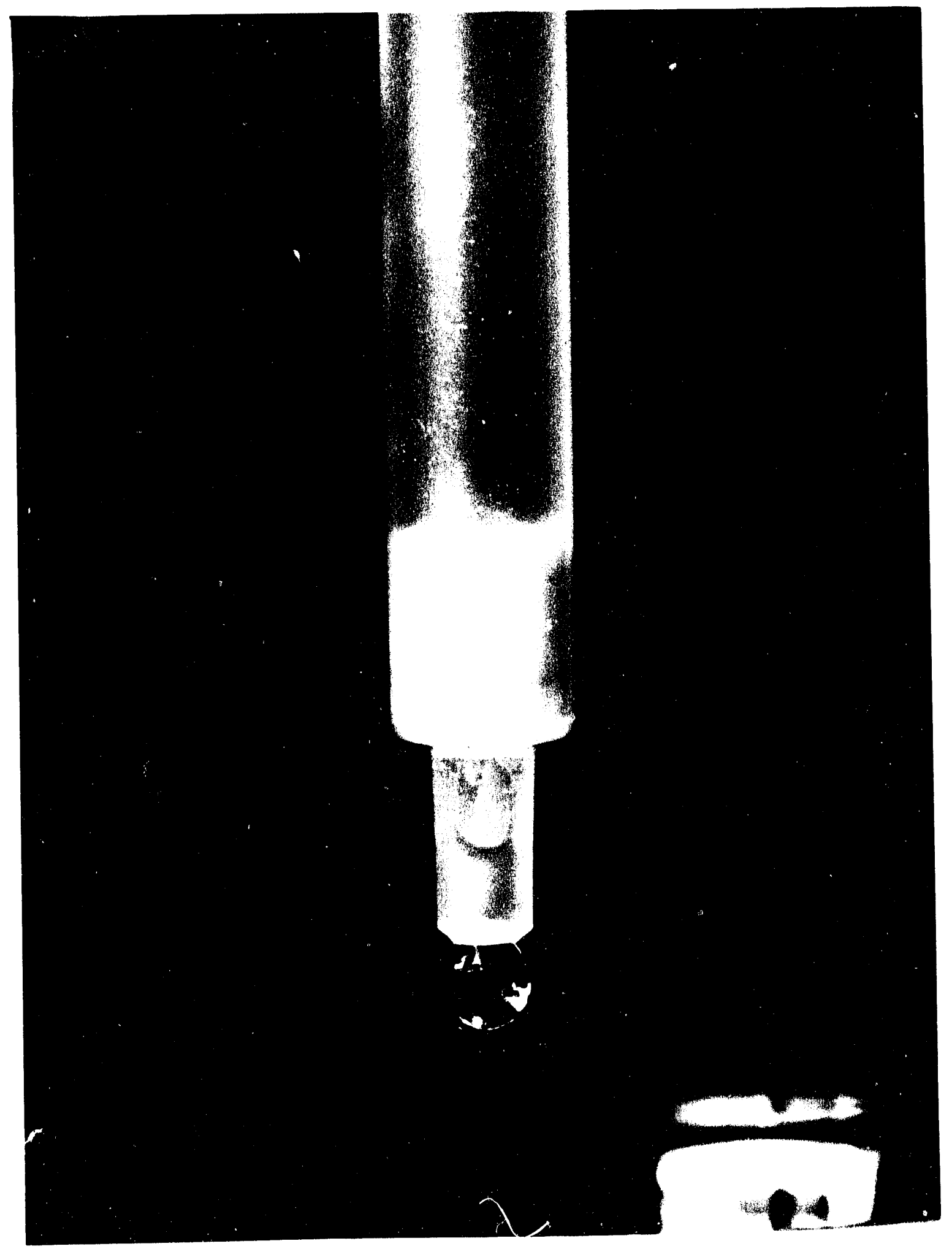


Figure 3. Photograph of Arsenazo III solution passing through SPE column, and extraction of colored complex. 


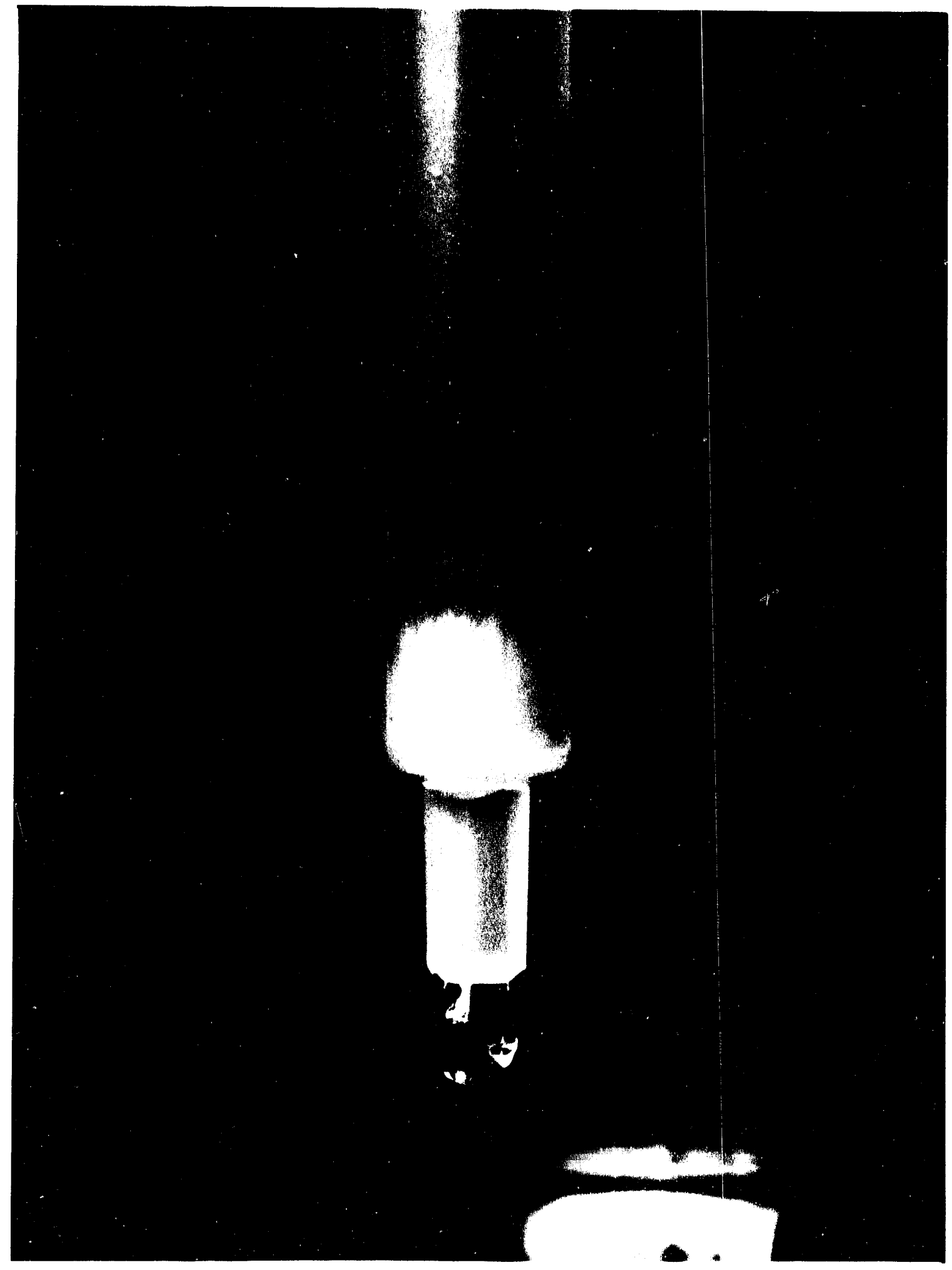


13

Figure 4. Elution step of solid phase extraction. 


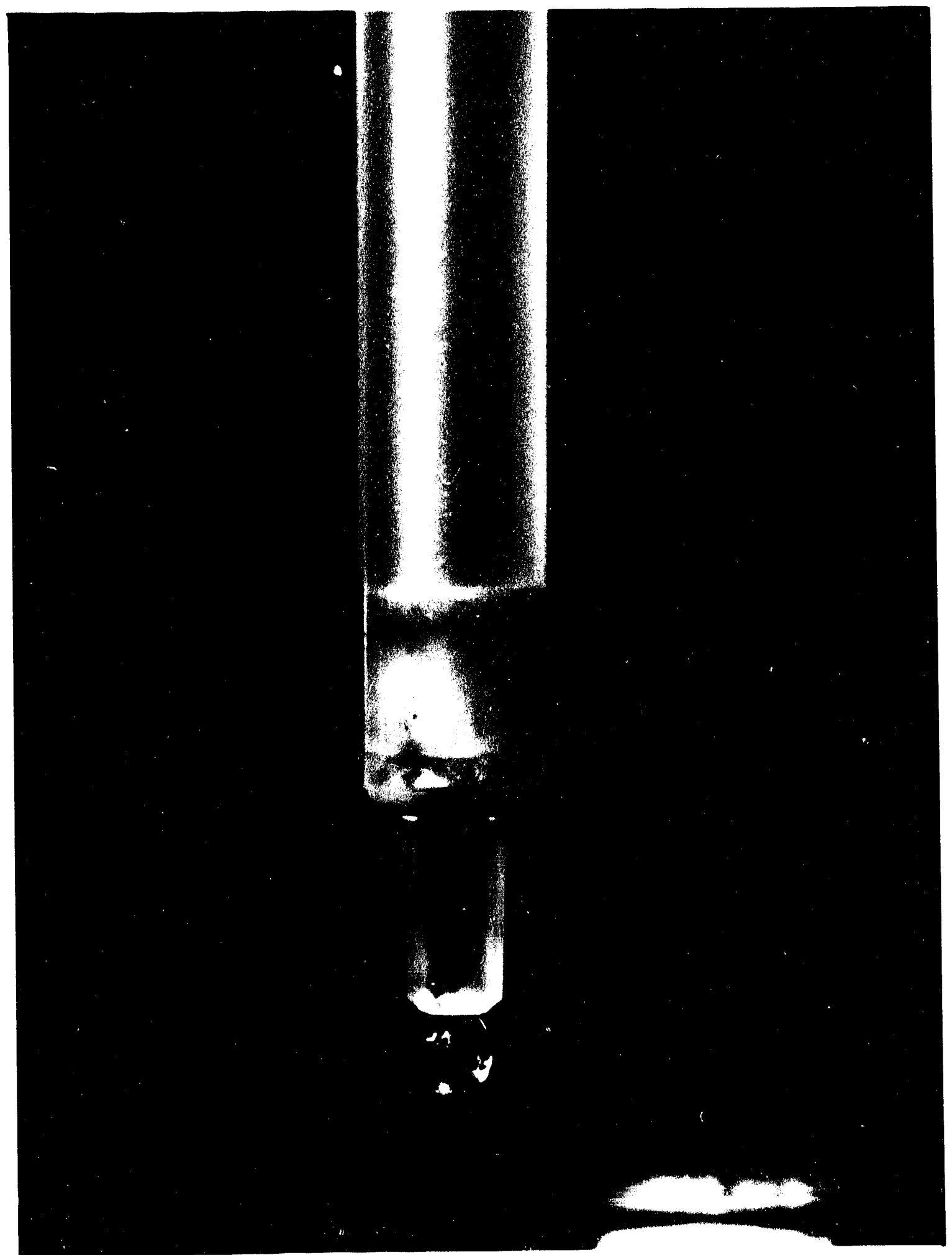


required for automation, SPE is amenable to on-line separations and concentrations.

M. Hennion described an on-line SPE concentration using a PRP-1 precolumn that could be switched into sequence with a $\mathrm{C}_{18}$ analytical column (13). The analytes were first concentrated out of a $10 \mathrm{ml}$ aqueous sample, then the samples are eluted off the precolumn and separated on the second column with an acetonitrile/water eluent.

M. Chiba and others (16) constructed an automated SPE/HPLC system for the cleanup, preconcentration, and analysis of several pesticides. Eight pesticides were concentrated on a commercial $C_{18}$ SPE column and eluted with acetonitrile. These concentrated pesticides were than analyzed by HPLC. Recoveries ranged from 84-93\% with a standard deviation of less than 5\%. Total analysis time was 90 minutes.

R. Majors and K. Fogelman (14) described a commercially available on-line SPE with a gas chromatograph. This instrument allows for an automatic cleanup and extraction with the SPE adsorbent of choice.

In $1991 \mathrm{~J}$. Sun and J. S. Fritz (40) demonstrated a method for the on-line extraction of basic compounds by placing a sulfonated polymeric resin in the injection port of a gas chromatograph. This sulfonated resin, placed in the 
injection liner and held in place with glass wool, extracted basic compounds from a sample solution while letting neutrals pass. The abstraction of basic compounds was essentially quantitative, but as yet no method has been developed for the elution of the adsorbed bases.

In similar work also by Fritz and Sun (42), a mercuric resin for the selective abstraction of mercaptans was developed. This resin was also placed in the split liner of a GC. It retained mercaptans, allowing other organic sulfur and non-sulfur compounds to pass.

There are many factors to consider when choosing a resin for SPE. G. Junk and J. J. Richard (17) pointed out the advantages of a smaller resin particle size in the concentration of pesticides and polycyclic aromatics. At this time (1988) polymeric resins were limited in diameter in comparison to silica based resins. Commercially available polymeric resins were much larger than their silica counterparts. They found that the smaller particle size of the silica resin made for a more efficient solid phase extractant. It facilitated higher flow rates and needed smaller volumes of organic solvent for complete elution. It was also more adaptable to hand-held syringes, making on site extractions possible. However, recent advances in the processes used in the preparation of polymeric resins by such 
companies as Sarasep, Inc. (Santa Clara, CA), have made polymeric resins available in particle sizes comparable to those of porous silica.

Chiba and coworkers have studied the use of $\mathrm{C}_{18}$ silica columns for the automated extraction of pesticides from aqueous samples (45). They studied the effect on the extraction of the flow rate of sample through the sorbent bed, the amount if sorbent needed, and the sorbent packing particle diameter. For this application, they found a flow rate of $10 \mathrm{ml} / \mathrm{min}$ could be used, and the size and amount of resin varied dependent upon the desired application.

In another paper by G. Junk and coworkers (15), the interferences encountered when silica-based $c_{18}$ bonded phase columns were used for SPE were studied. They found a large number of interferences in commercially available SPE columns. They found low level impurities could be extracted from the $c_{18}$-bonded porous silica, the polypropylene housing, and the polyethylene frits used to hold the resin in the tube. The intensity of the peaks is not only dependent upon the commercial column used, but also the eluting solvent used and its ability to extract low level impurities from polyethylene and polypropylene. A large number of silanol interferences were observed that could be attributed to hydrolysis of the $\mathrm{c}_{18}$-bonded phase silica. It was suggested 
18

that a solid phase more inert than porous silica would be useful.

SPE has demonstrated usefulness other than preconcentration and cleanup. Quite often isolation of a specific analyte can be achieved or, in other cases, fractionation of complex samples. M. Mills and E. Thurman at the University of Kansas used a mixed mode SPE scheme in the isolation of triazine metabolites from soil and aquifer sediments (44). The analytes were first extracted with methanol/water from the soil and then concentrated on a $c_{18}$ column. They were then eluted with ethyl acetate. After this, the small volume eluate was passed through an anion exchange SPE column to remove coeluted humic substances. Recoveries from these quite difficult samples were $\approx 75 \%$, with standard deviations of $\approx 15 \%$.

The most widely used general technique used for the identification of volatile organic compounds in complex mixtures is GC/MS. In many cases, the gas chromatograph gives inefficient separation. In these instances, some type of group separation or fractionation is performed prior to analysis to simplify the mixture and gain some information about the chemical nature of the components. Liquid/liquid techniques are generally used in these instances $(23,24,25)$. A 1981 paper by Svec and Colgrove (22) detailed a method for 
19

fractionation of strong and weak acids, bases, polar and nonpolar compounds, aldehydes, and ketones by liquid/liquid extraction. This fractionation was achieved by performing multiple extractions from aqueous to methylene chloride with pH adjustments and Girard's Reagent-T for the ketones. While this was the most comprehensive study of liquid-liquid extraction for fractionation, the group separations reported were often incomplete, and recoveries ranged from 40-80\% with an average of around $70 \%$ for most compounds. In contrast, the SPE method described here is faster, generates much less waste, and exhibits recoveries of $90 \%$ or better for compounds present at 10-40 ppm.

The EPA has directed an SPE method for the fractionation of nonpolar organic toxicants in aqueous samples using an methanol-water elution gradient (26).

In an EPA report put out in 1989 (27), V. Lopez-Avila and coworkers used a variety of SPE columns including fluorosil, silica gel, and alumina to fractionate a variety of PCB's and phenolic compounds.

F. Ulberth and E. Achs (28) used a commercially available silica-based column, CHROMABOND SA, to fractionate the methyl esters of a fatty acid mixture. Fractionation was achieved by varying solvent polarity. The recoveries were quite good (in most instances 95 - 100\%). 
N. Theobald (29) demonstrated the ability to fractionate petroleum hydrocarbons using reversed phase $\left(C_{18}\right)$ and normal phase $\left(\mathrm{SiO}_{2}\right)$ SPE columns.

Aldridge and Oates (30) did similar work separating aliphatic and aromatic hydrocarbons usins a $\mathrm{C}_{18}$ column. Altiough fractionation was not complete, it was adequate for the sample mixtures studied which included gasoline, diesel fuel, and samples taken from a suspected arson sit?

Thompson and coworkers (36) performed a separation of basic and neutral drug fractions on a silica based copolymeric column.

J. R. Benson devised a scheme for the concentration of basic drugs on polymeric columns (37). Hexanesulfonic acid was used as an ion-pairing agent to extract amphetamine and methamphetamine from urine samples. Recoveries in both cases averaged $>90 \%$ with a standard deviation of $6 \%$.

Fritz and Kaczvinsky (38) demonstrated a method for the extraction of basic organic compounds from aqueous solution. The bases were taken up as cations on $2.5 \mathrm{~g}$ of sulfonated polymeric resin. Recoveries were quite good, generally 90 100\%, but large volumes of ammonia in methanol (40 ml) were needed for elution of the bases due to the large volume of resin needed. This large elution volume necessitated a concentration step prior to analysis. 
21

Parry and coworkers at supelco (39) used a weak cation exchange silica resin for solid phase extraction of four catecholamines from blood plasma. The amines were eluted from the resin $\mathrm{HClO}_{4}$ in water and analyzed by HPLC. Recoveries of the basic drugs were all 90 - $100 \%$ with standard deviations of 5-10\% for three trials.

In 1975, Fritz, Chang, and Chriswell first demonstrated the use of polymeric anion exchange resin for the solid phase extraction of phenols from water as phenolate anions (41). Sodium hydrosulfite was added to the solution in order to minimize the oxidation of phenol in basic solution. The concentrated phenols were eluted by first passing aqueous $\mathrm{HCl}$ through the resin to convert the phenols into their molecular form and then eluting with methylene chloride and acetone. Once again a rather large volume of eluent was needed, so a concentration step in which excess slvent was boiled off was performed. Recoveries of phenols from tap water were 90 $100 \%$.

A. DiCorcia and coworkers (31) have previously attempted a group separation of acids and neutrals. In this work a separation was performed using graphitized C black as an SPE adsorbent. Two modes of adsorption were achieved by utilizing naturally occurring positively charged centers for the electrostatic adsorption of anions, and hydrophobic 
22

attraction for adsorption of neutrals. A rather large amount of adsorbent (250 $\mathrm{mg})$ and eluent are needed, due to the fact that $C$ black is a rather inefficient resin.

N. Yamaga and coworkers (32) have demonstrated a liquidliquid method for the group separation of bile acids. They used a variety of internal standards added to the mixture in order to quantitate the acids present in each fraction.

J. Street and others (33) concentrated a mixture of bile acids on a $\mathrm{C}_{18}$ SPE column. They fractioned the samples into 3 groups: unconjugated, glycine- and taurine- conjugated bile acids by varying the polarity of the eluent (ethanol/ chloroform/ water mixture buffered with acetate).

\section{PE with Resin Impregnated Membranes}

One of the biggest disadvantages of SPE is the formation of channels through the resin bed at large sample volumes. As more and more liquid is forced through the resin bed at relatively high pressure, the solution starts to form favored pathways, or "channels", through the column. This leads to decreased extraction efficiency due to a decrease in mass transfer efficiency and significantly lowers the effective capacity of the resin. The membrane technology, first introduced by Markell and Hagen of 3M in 1990 (10), tackles this problem. They embedded $c_{18}$ resin particles in a polytetrafluoroethylene (PTFE) fibril network, thus holding 
the resin firmly in place. The PTFE network consists of less than $10 \%$ of the total weight of the membrane, leaving $90 \%$ of the membrane available for adsorption sites. By impregnating the resin in a membrane, the individual resin particles are stabilized, thus inhibiting channel formation. This allows for higher flow rates and larger back pressures. In addition, it ensures that the entire resin bed will be put to use, thus maximizing the capacity of the resin. This technology significantly decreases solvent consumption. To extract a pesticide analyte from $4 \mathrm{~L}$ of aqueous solution using liquid-liquid extraction, roughly comparable volumes of organic solvent must be used. In comparison, using a $\mathrm{Cl} 8$ empore disk for the extraction of pesticides from water (Barcelo and coworkers, ref. 11), a 4 liter sample containing $1-4000 \mu \mathrm{g}$ of pesticide was concentrated on a extraction disk by passing the sample through the disk. The analyte was then eluted with $20 \mathrm{~mL}$ of methanol.

SPE disks have been used in the sample preparation steps in a number of methods for water analysis in environmental laboratories (19). These disks have shown significant improvements in methodology for water analysis. Higher throughput leads to shorter analysis time and larger concentration factors. The stabilized resin particles resist 
the channeling effects suffered by loose resins at high flow rates and large volumes. Senseman, et al. (12) have demonstrated a significant advantage in pesticide stability when stored on extraction disks when compared to aqueous storage. A large variety of pesticides were studied with storage periods of up to 180 days and storage temperatures of $-20^{\circ} \mathrm{C}$ to $4^{\circ} \mathrm{C}$. They reported better recoveries of pesticides using the membrane for storage in all cases. Particularly unstable pesticides, such as Captan, showed markedly improved results when stored on the disk rather than in solution. It has also been suggested (19) that these disks be used for onsite sample collection, with the sample loaded disk being transported to the laboratory for analysis, rather than collecting large liquid samples and transporting these solutions to the laboratory.

While there are numerous examples of the use of SPE disks in the extraction of hydrophobic analytes, low recoveries are often obtained when more polar, water soluble analytes such as phenols are studied. Several techniques have been tried in an attempt to increase recoveries, including $\mathrm{Ph}$ adjustment to neutralize the phenol, addition of a salt to "salt out" the phenol, alternative sorbents, larger sorbent beds, ion pairing, and smaller sample volumes. 
Sun and Fritz (6) have shown that resins made more hydrophilic by chemical derivatisation adsorb polar molecules more effectively than 018 particles. This modified resin allows for a more intimate contact to occur between the bulk solution and the resin surface. 
PART I.

BOLID-PHABE EXTRACTION OF PHENOLS UBING MEMBRANES LOADED WITH MODIFIED POLYMERIC RE8INS. 


\section{INTRODUCTION}

In 1989 a new family of materials for solid phase extraction (SPE) was introduced (10). This is a membrane, or disk of polytetrafluoroethylene (PTFE) fibrils impregnated with small 5-8 $\mu \mathrm{m}$ particles of adsorbing material such as C18 silica or poly-styrene divinyl benzene (PS/DVB). The mass of these membranes, developed and manufactured by the 3M company (St. Paul, MN, USA), is $90 \%$ resin particles, with the PTFE network being only 10\%. This fibril network stabilizes the resin bed, prevents channeling, and improves mass transfer. An electron micrograph of such a membrane is shown in Figure 5. As illustrated, the bulk of the membrane is resin. Flow rates as fast as $200 \mathrm{ml} / \mathrm{min}$ are possible, yet uptake of organic solutes is efficient because of fast kinetics. These faster kinetics are a result of smaller particle size, close and uniform packing, and elimination of channeling when compared to standard loose packing SPE.

The discharge of phenolic compounds by industry and municipalities is of great concern to the public (20). There is great interest in the determination of phenols, as illustrated by the large amount of papers generated on the subject. The majority of the analyses, including the traditional E.P.A. method, call for liquid-liquid extraction. This is usually performed by acidifying the aqueous sample 
Figure 5. Electron micrograph of acetyl membrane. 


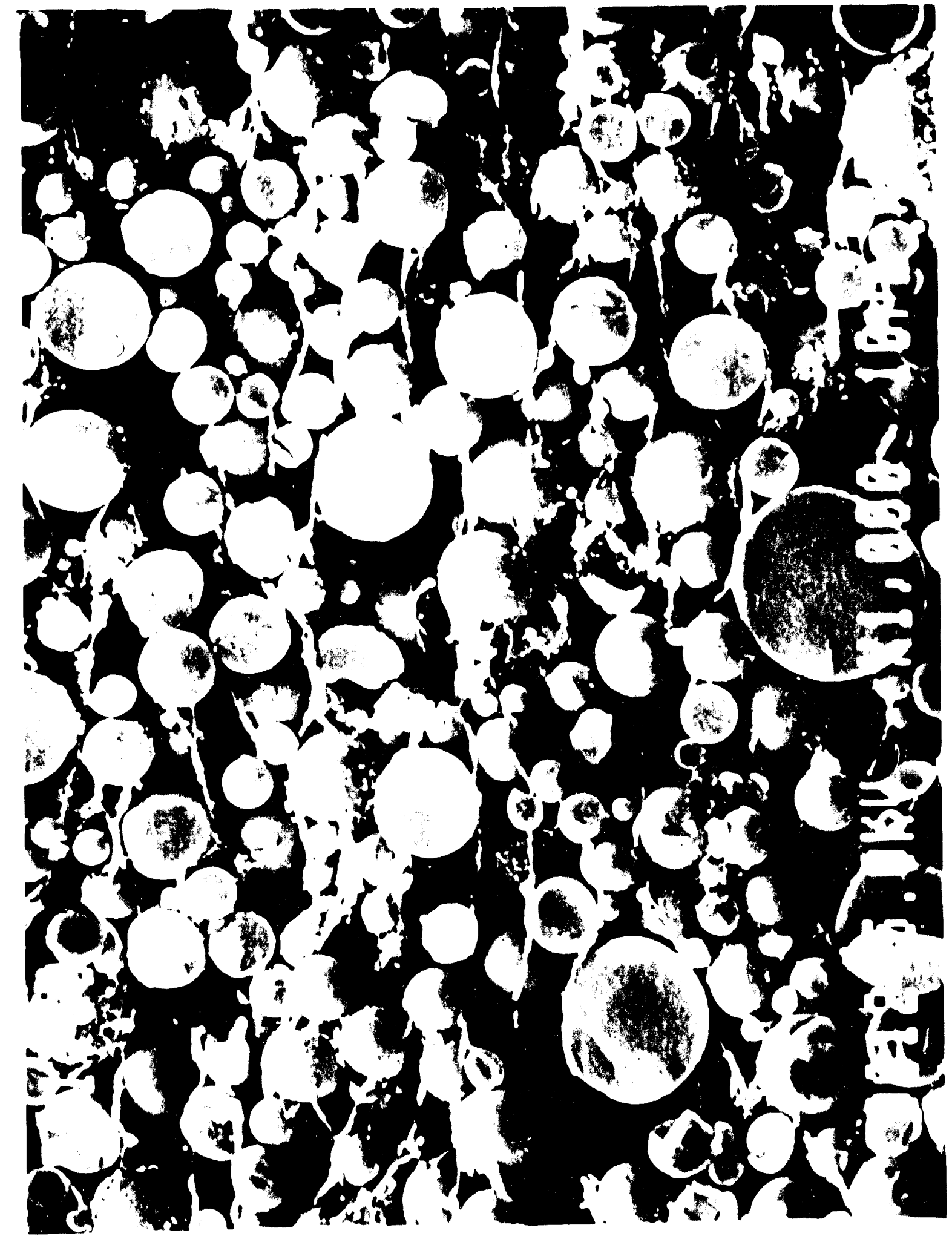


containing the phenols and extracting with a roughly equal volume of methylene chloride. This is followed by a concentration step (boiling down) and then analysis by GC. Solid Phase Extraction is clearly a simpler and cleaner approach, and has been suggested by some (21). Traditional SPE supports $\left(S i-C_{18}\right.$, PS/DVB, etc.), show good recoveries of some phenols, but are generally too hydrophobic to show good extraction efficiencies of phenol itself and others.

A 1990 paper by sun and Fritz (18) introduced a number of chemically modified polymeric resins for high-performance liquid chromatography. Among these resins was an acetyl derivatized resin which displayed increased hydrophylicity. In a 1992 paper (6), Sun and Fritz demonstrated the usefulness of this resin for SPE. In comparison with silica $c_{18}$ and underivatized resins, the acetyl resin was vastly superior in its ability to extract polar molecules from aqueous samples.

This work explores the use of resins impregnated with acetyl derivatized resins for the extraction of phenols from aqueous samples. The increased hydrophylicity of the resin encourages intimate surface contact between resin surface and solution and makes for a more efficient extraction of the more polar phenols. 
31

The use of the membrane further improves the extraction. A novel method of incorporating these membranes into SPE tubes is also introduced. 


\section{EXPERIMENTAL}

\section{Apparatus}

Solid-phase extractions were performed using the laboratory-made apparatus shown in Figure 6 . It consisted of a $30 \mathrm{ml}$ glass reservoir connected by a small adapter to the SPE column containing the membrane plug. The columns were connected to the reservoir by an adapter (Alltech Assoc., Deerfield, IL, U.S.A.). The SPE columns were obtained from Varian (Harbor City, CA, U.S.A.). The membrane was held in place above and below by polyethylene frits. The top of the reservoir has a ground glass joint which may be connected to a source of constant adjustable air pressure, which was used to control the flow rate of the liquid sample through the membrane.

A Hewlett Packard 5880 gas chromatograph with a flame ionization detector and an HP $5880 \mathrm{~A}$ series 4 integrator was used to analyze ali phenols. An HP 7673A autoinjector was used to introduce a $2 \mu l$ sample onto the column. Separations were performed using a fused silica capillary SPB-1 column (Supelco, Inc., Bellefonte, PA, U.S.A.). 


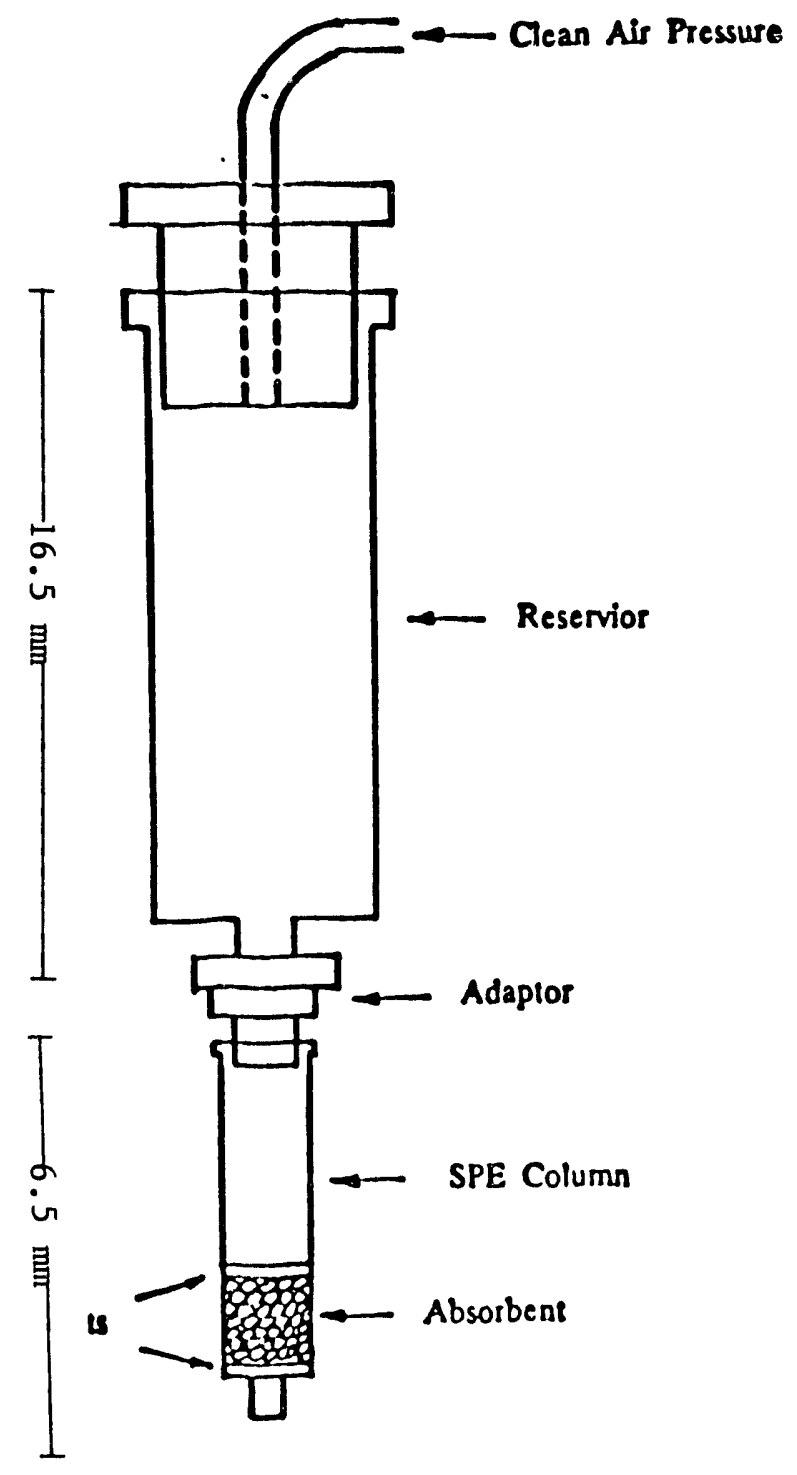

Figure 6. Laboratory made SPE apparatus. 


\section{Reagents and Chemicals}

The reagents and chemicals used for the derivatisation were reagent grade and were dried by molecular sieves. They were obtained from the Aldrich Chemical Company (Milwaukee, WI, I.S.A.). The phenols used in the experiment were analytical standards obtained from supelco, Inc. (Bellefonte, PA) - Laboratory distilled water was further deionized by a Barnstead Nanopure II system (Sybron Barnstead, Boston, MA, U.S.A) •

\section{Preparation of Modified Resins}

The resin used in this experiment was a PS/DVB resin obtained from SRP 80 (Sarasep, Inc., Santa Clara, CA). The structure of this porous polymeric resin is shown in Figure 1 of the Introduction. It is spherical and has an average diameter of $8 \mu \mathrm{m}$ and a surface area of about $400 \mathrm{~m}^{2} / \mathrm{g}$. The resin was rinsed with methanol, acetonitrile, and acetone before derivitisation.

The reaction used in the introduction of the acetyl functional group onto the resin was first described by sun and Fritz (18) and is shown below:

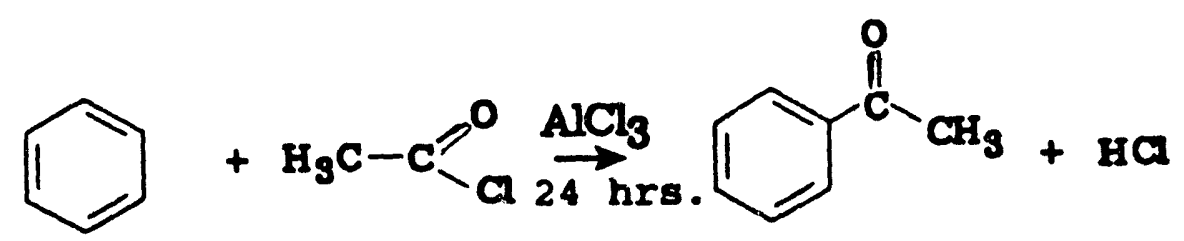


The preparation consists of a simple one-step reaction. 5.1 of resin was suspended in $30 \mathrm{ml}$ of carbon disulfide. Anhydrous aluminum chloride $(9.5 \mathrm{~g})$ was then added as catalyst. To this mixture, $5.5 \mathrm{~g}$ of acetyl chloride was added dropwise. This mixture was lightly stirred and allowed to react at $50^{\circ} \mathrm{C}$ for 24 hours. The product was then poured into ice water to quench the reaction. This mixture was then filtered over a medium glass frit and rinsed with acetone, methanol, and water. The presence of the carbonyl group was confirmed by the presence of a strong FTIR band at $1690 \mathrm{~cm}^{-1}$. A physical indication that the hydrophilic group has been introduced can be seen by adding a small amount of resin to water. The underivatized resin forms clumps and floats on the surface of the water, while the hydrophilic resin breaks apart easily and forms a suspension in the water. These reaction conditions give an acetyl capacity of $1.2 \mathrm{mmol} / \mathrm{g}$ as determined by oxygen analysis.

\section{Procedure for $\mathrm{BPE}$}

Membranes containing the acetyl resin were prepared by the $3 \mathrm{M}$ company (St. Paul, MN, U.S.A.) using resin prepared here at Iowa State University. Two types were prepared, one approximately $1 \mathrm{~mm}$ thick, and another approximately $3 \mathrm{~mm}$ thick. In this experiment only the thicker membrane was tested. 
A membrane circle with a diameter of about $7.5 \mathrm{~mm}$ was cut out of the membrane sheet. This plug was then forced into the SPE column (7 mm i.d. by $55 \mathrm{~mm}$ ) with $20 \mu \mathrm{m}$ polyethylene frits above and below for support. Physical pressure was then applied to the membrane, causing it to form a seal with the edges of the SPE tube. This column was then attached to the SPE apparatus shown in Figure 6.

The sample solution was prepared by adding a standard solution of phenols (50 ppm each) in methanol to 20 to $30 \mathrm{ml}$ of water solution so that the final concentration was approximately $0.4 \mathrm{ppm}$ each. The $\mathrm{Ph}$ of the solution was lowered to approximately 2 with sulfuric acid to repress ionization of the more acidic phenols.

Prior to each use a small amount of methanol (ca. $1 \mathrm{ml}$ ) was added to the column to "prewet" the membrane. Without allowing the membrane to dry, the sample solution was passed through at a flow rate of $2 \mathrm{ml} / \mathrm{min}$ by applying a pressure of 15 p.s.i. The column was then rinsed with $2 \mathrm{ml}$ of distilled water.

The phenols were then eluted from the column with 0.75 $\mathrm{ml}$ of either methanol or methylene chloride. This gives a concentration factor of approx. 25 to 40 . Either eluant was found to be sufficient to completely wash off the extracted phenols. The eluate was collected in a $1.8 \mathrm{ml}$ GC vial, to 
which $0.1 \mathrm{ml}$ of internal standard (500 ppm quinoxaline) was added. The vial was then capped and mixed with an orbital stirrer.

A $2 \mu l$ aliquot of the sample mixture was then injected into the gas chromatograph with a split ratio of $1: 40$. Helium carrier gas was used at a flow rate of $15 \mathrm{ml} / \mathrm{min}$. The oven temperature was held initially at $65^{\circ} \mathrm{C}$ for 2 minutes, and then ramped at $15^{\circ} \mathrm{C} / \mathrm{min}$ to a final temperature of $250^{\circ} \mathrm{C}$. Detection was by flame ionization detector. Identification of phenols was based on retention time comparison of sample with standard. The recoveries were calculated by comparison of relative peak height to that of a standard consisting of the original methanol solution with internal standard added not subjected to SPE. To obtain an average recovery, a minimum of 3 trials was used in all cases. 


\section{RESULTB AND DIBCUSBION}

The most common mode of SPE in chemical analysis is with small tubes or cartridges filled with loose polymeric resin or bonded-phase silica particles. Usually a bed height of > $1 \mathrm{~cm}$ is used to ensure good retention of the desired sample compounds. However, this necessitates a relatively large volume of solvent $(>1 \mathrm{ml})$ to quantitatively elute the adsorbed compounds. By packing the tubes with membrane disks 5-7 $\mathrm{mm}$ in diameter, more efficient elution is possible due to the shorter resin bed height. It was found that elution volumes of $0.25 \mathrm{ml}$ were sufficient to elute the phenols quantitatively. The reason for this is that in the membrane the resin particles are smaller $(5-8 \mu \mathrm{m}$ vs.40 $\mu \mathrm{m})$, more closely packed, and more evenly dispersed throughout the membrane when compared to loose packed resins. Since the particles are immobilized, it is possible to avoid the channelling that would be likely in a tube containing a shorter loose resin bed.

Earlier work done by Dr. Jeff Sun (Iowa state University, unpublished) had focussed on trying to incorporate these membranes into SPE tubes to take advantage of the physical stability of the resin when embedded in the membrane. Six of the normal $1 \mathrm{~mm}$ thick membranes were stacked within the SPE tube, and held in place with frits. 
Results were good for some runs, but erratic. Tests with an azo dye, pyridylazonaphthol, showed that channeling occurred on the outer rim of the disks. It took great care to ensure that the disks fit tightly in the SPE tube.

It appeared that a single, thicker membrane would work well for SPE in a small column. A membrane of thickness of about $3.5 \mathrm{~mm}$ was prepared for us by the $3 \mathrm{M}$ company. A plug slightly larger ( $7 \mathrm{~mm}$ diameter vs. $6.5 \mathrm{~mm}$ ) than the diameter of the SPE tube was then cut out of the membrane sheet with a cork borer. This plug is shown in Figure 7. The plug was gently forced into the SPE tube with PTFE frits above and below for support. The excess membrane served to form a tight seal with the edge of the tube. Tests with an azo dye showed no channeling around the outer edges, thus solving the problems seen with the thinner membranes.

\section{Deionized water}

The first tests were run on $30 \mathrm{Ml}$ deionized water samples spiked with phenol standards and acidified with $\mathrm{H}_{2} \mathrm{SO}_{4}$. The samples were passed through the acetyl membrane in the SPE column at a flow rate of $1 \mathrm{~mL} / \mathrm{min}$. The phenols were adsorbed onto the resin by hydrophobic interactions and subsequently eluted with methanol or methylene chloride. Tests showed an elution volume of $0.25 \mathrm{~mL}$ of either solvent was adequate to elute $>95 \%$ of the phenols. In the GC step of 
Figure 7. Photograph of acetyl membrane plug. 


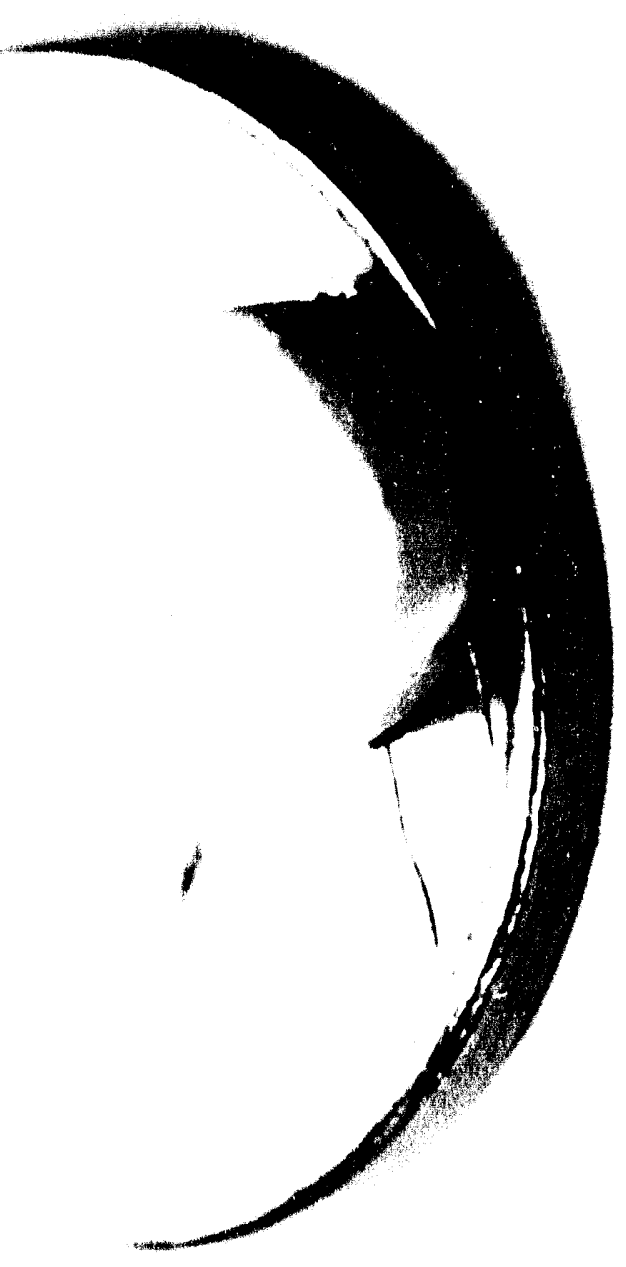


the analysis, however, a volume of $\approx 0.75 \mathrm{~mL}$ was needed in the vial in order for the needle in the autoinjector to reach the solution, so elutions were performed using $0.75 \mathrm{ml}$ of methanol. An example of the chromatogram obtained is shown in Figure 8 . The average recoveries $(n=3)$ of a large number of phenols is given in Table $I$. Recoveries of the phenols were quite good, with most recoveries in the 95 to 1008 range.

\section{Tap and River Waters}

Realizing that the deionized water samples may be more ideal and not representative of the types of samples found in the real world, similar experiments were run on spiked tap and river water samples. Samples of both types were spiked to phenol concentrations of $0.4 \mathrm{ppm}$ and $20 \mathrm{ml}$ of these solutions were acidified and passed through the membrane. Once again the phenols were eluted with methylene chloride and analyzed by GC. An example of the chromatogram obtained is shown in Figure 9. Recoveries of the phenols from these real world samples were also quite good, with the exception of 2-methyl-4,6-dinitrophenol in tap water, the recoveries were generally $>90 \%$. The recoveries are reported in Tables II and III. 


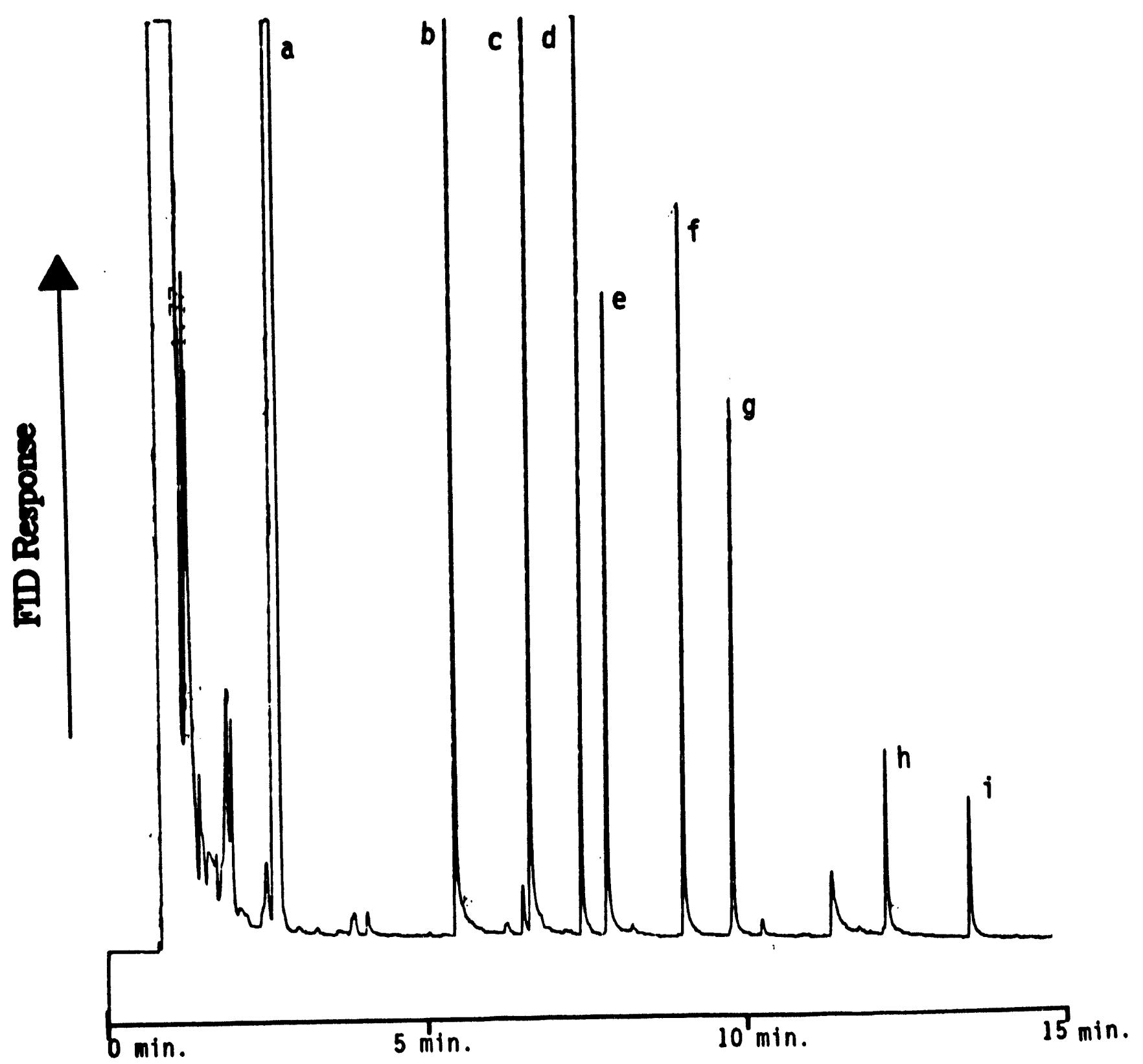

Figure 8. Gas chromatogram of phenols concentrated from deionized water solution. 
44

Table I. Percent recoveries of phenols from delonized water on acetyl membrane. Elution with $0.75 \mathrm{~mL}$ of methanol. $(n=3)$

\begin{tabular}{|c|c|c|}
\hline Analyte & \&ecovery & std. Dev. \\
\hline Phenol & 99 & 2 \\
\hline 3-Methylphenol & 95 & 1 \\
\hline 2-Nitrophenol & 100 & 4 \\
\hline 2,4-Dichlorophenol & 98 & 0 \\
\hline 4-Chloro-3-methyl phenol & 98 & 0 \\
\hline $2,4,6$-Trichlorophenol & 102 & 0.5 \\
\hline Pentachlorophenol & 100 & 2 \\
\hline 2-Chlorophenol & 99 & 2 \\
\hline 2-Methylphenol & 99 & 4 \\
\hline 4-Methylphenol & 97 & 5 \\
\hline 2,4-Dimethylphenol & 97 & 5 \\
\hline 2,6-Dichlorophenol & 100 & 5 \\
\hline $2,4,5$-Trichlorophenol & 96 & 5 \\
\hline $2,3,4,6$-Tetrachlorophenol & 99 & 3 \\
\hline 2-sec-Butyl-4,6-dinitrophenol & 101 & 4 \\
\hline 2-Methyl-4,6-dinitrophenol & 94 & 2 \\
\hline
\end{tabular}




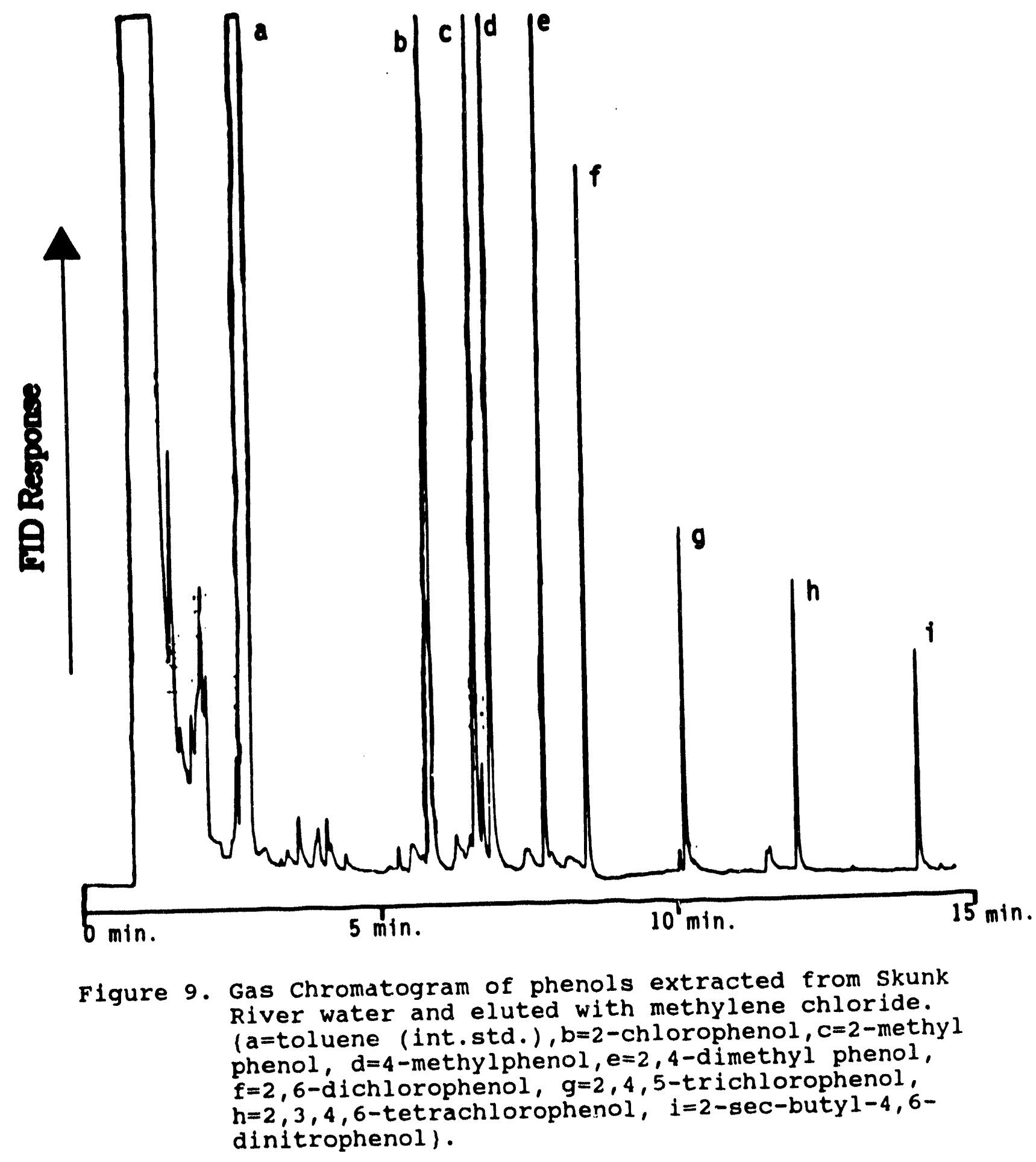


Table II. Percent recoveries of phenols from tap water using acetyl membrane.

\begin{tabular}{lcc} 
Analyte & f Recovery & Std. Dev \\
\hline Phenol & 96 & 2 \\
3-Methylphenol & 93 & 2 \\
2-Nitrophenol & 102 & 3 \\
2,4-Dichlorophenol & 97 & 3 \\
4-Chloro-3-methyl phenol & 85 & 2 \\
2,4,6-Trichlorophenol & 98 & 3 \\
Pentachlorophenol & 92 & 1 \\
2-Chlorophenol & 106 & 4 \\
2-Methylphenol & 100 & 3 \\
4-Methylphenol & 98 & 2 \\
2,4-Dimethylphenol & 94 & 2 \\
2,6-Dichlorophenol & 95 & 5 \\
2,4,5-Trichlorophenol & 95 & 4 \\
2,3,4,6-Tetrachlorophenol & 95 & 5 \\
2-sec-Butyl-4,6-dinitrophenol & 95 & 2 \\
2-Methyl-4,6-dinitrophenol & 95 & 2 \\
\hline
\end{tabular}


Table III. Percent recoveries of phenols from skunk River water on acetyl membrane. $(n=3)$

\begin{tabular}{|c|c|c|}
\hline Analyte & \& Recovery & std. Dev. \\
\hline Phenol & 97 & 3 \\
\hline 3-Methylphenol & 92 & 4 \\
\hline 2-Nitrophenol & 98 & 1 \\
\hline 2,4-Dichlorophenol & 97 & 2 \\
\hline 4-Chloro-3-methyl phenol & 93 & 4 \\
\hline $2,4,6$-Trichlorophenol & 101 & 0 \\
\hline Pentachlorophenol & 100 & 4 \\
\hline 2-Chlorophenol & 103 & 2 \\
\hline 2-Methylphenol & 100 & 1 \\
\hline 4-Methylphenol & 98 & 4 \\
\hline 2,4-Dimethylphenol & 103 & 5 \\
\hline 2,6-Dichlorophenol & 104 & 6 \\
\hline $2,4,5-T r i c h l o r o p h e n o l$ & 107 & 3 \\
\hline $2,3,4,6$-Tetrachlorophenol & 101 & 0.5 \\
\hline 2-sec-Butyl-4,6-dinitrophenol & 101 & 2 \\
\hline 2-Methyl-4,6-dinitrophenol & 95 & 2 \\
\hline
\end{tabular}




\section{CONCLUSION}

Phenols in the low ppm concentration can be effectively concentrated from aqueous samples by SPE with a membrane loaded with acetyl PS/DVB resin particles. Larger sample volumes can be passed through these membranes with no obvious channeling effects. Very small volumes of organic solvent are needed to elute the adsorbed phenols, leading to very large concentration factors. This technique appears to be superior to other standard methods of phenol concentration and cleanup, as it is rugged, quick, and efficient. 
49

PART II.

ION EXCHANGE PRECONCENTRATION AND GROUP

GEPARATION OF BABIC AND NEUTRAL ORGANIC

COMPOUNDB 


\section{INTRODUCTION}

Solid-Phase Extraction (SPE) is fast becoming the preferred technique for analytical preconcentration (1). It uses much smaller amounts of organic solvents than liquidliquid extraction. SPE is easily automated and is capable of obtaining very high concentration factors.

SPE is usually carried out with a very small tube (or column) packed with a spherical solid of small particle size and high surface area. It can be thought of as low performance liquid chromatography. Maximum retention is desired for the substances of interest with minimum retention of the other sample materials. The adsorbed substance can be subsequently eluted by a very small volume of an organic solvent or other suitable solvent.

The use of a short ion-exchange precolumn is well established $(38,39)$ to concentrate anions or cations prior to their separation and determination by ion chromatography. In the present work an ion-exchange resin with suitable properties is used to preconcentrate organic substances from aqueous samples and then separate them into two fractions: neutral and basic compounds. The key to this separation is adsorption in a dual mode: neutrals by a hydrophobic attractions at unreacted sites on the sulfonated resin, and 
51

protonated bases by electrostatic attraction at ion exchange sites. Figure 10 shows an illustration of this dual mode adsorption. The basis for this separation then is that neutral organic compounds are readily eluted from the SPE column by an organic solvent while basic compounds remain on the ion exchange resin as protonated cations. The latter can then be eluted by an organic solvent containing a base to neutralize the protonated solute cations. An outline for this 2-step elution is shown in Figure 11. This same principle has been used previously $(2,3,4)$, but not to any extent for simultaneous preconcentration and group separation. 


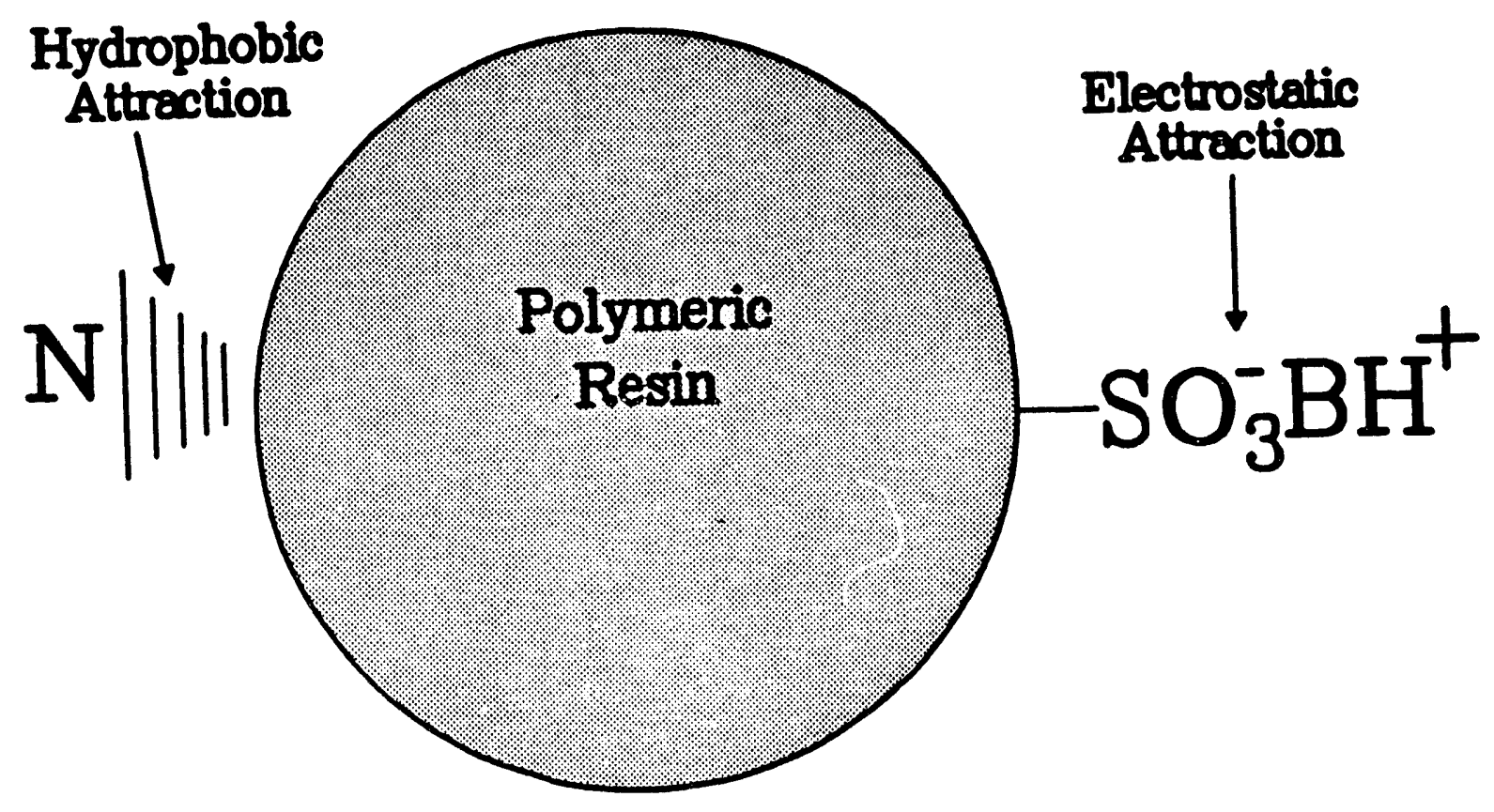

Figure 10. Diagram illustrating dual mode adsorption at single polymeric resin particle. 

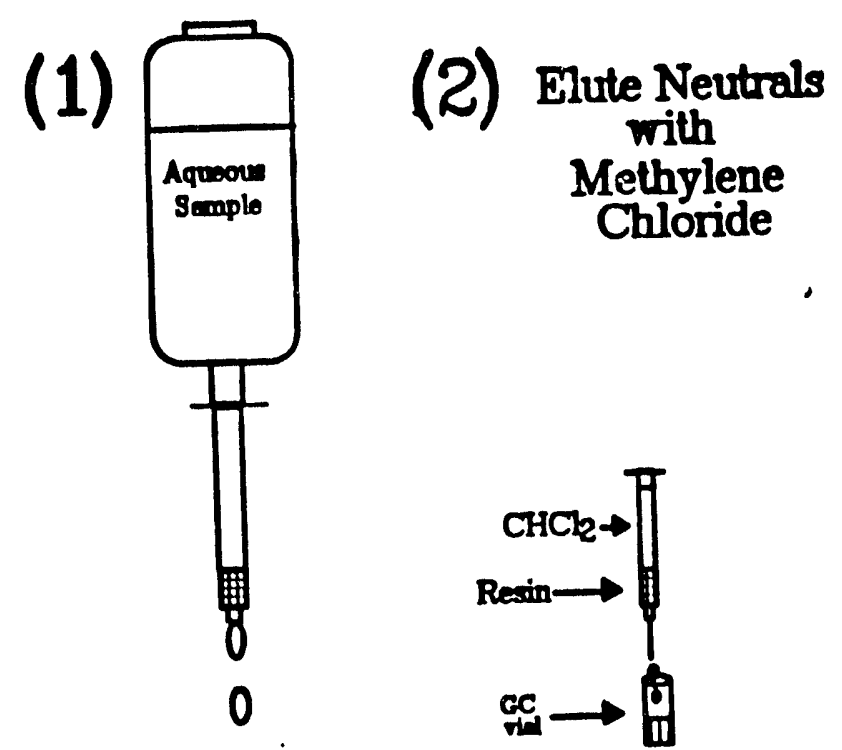

(3)

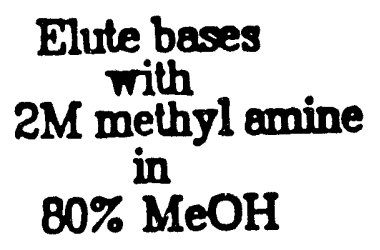

Experimental Procedure for preconcentration using cation exchenge resin

1. The $\mathrm{pH}$ of the equeous solution containing basic and neutral compouds was lowered with $\mathrm{HCl}$.

2. The column containing the cation exchange resin was pretreated with

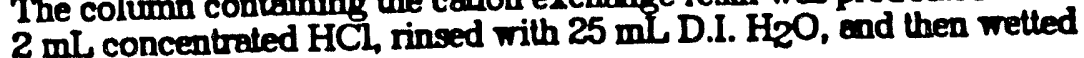
methanol.

3. The equeous somple solution is then pessed through the SPE column ad a flow rate of about $1 \mathrm{~mL} / \mathrm{min}$.

4. The neutrals are eluted first using $\mathrm{CHCl}_{2}$.

5. The beses are then eluted using Methyl amine or ammonia in methonol

Figure 11. Scheme for the group separation of acids and neutrals using anion exchange resin and 2 step elution process. 


\section{EXPERIMENTAL}

\section{Reagents and Chemicals}

The reagents used for the derivitisation of the ion exchange resins were of analytical grade. Reagents used and analytes studied in the solid phase extraction were $>998$ pure and used as obtained from Aldrich, Fisher, and Eastman-Kodak. Laboratory distilled water was further purified using a Barnstead Nanopure II System (Sybron Barnstead, Boston, MA, USA) •

The resins were prepared using a highly porous, crosslinked polystyrene resin, Amberchrome 161 (Supelco, Bellefonte PA, USA). This spherical resin has an average particle size of about $40 \mu \mathrm{m}$ and a surface area of about 720 $\mathrm{m}^{2} / \mathrm{g}$. An $8 \mu \mathrm{m}$ PS/DVB resin, SRP-80 (Sarasep, Inc., Santa Clara, CA), was used in the membranes for the separations of bases and neutrals in nonpolar solutions.

\section{Preparation of Cation Exchange Resin}

A simple one step reaction was used for the preparation of the cation exchange resin. The time of the sulfonation reaction was the parameter adjusted in order to control the extent of the reaction. The reaction was carried out at $0^{\circ} \mathrm{C}$ and is shown below:

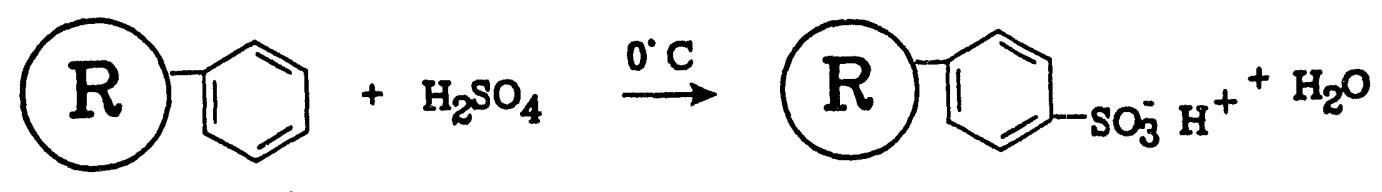

PS/DVB 
The procedure for the preparation of the sulfonated cation exchange resin follows. In an ice bath, 1 gram of resin was wetted with $1 \mathrm{~mL}$ of glacial acetic acid and stirred to form a slurry. To this slurry $25 \mathrm{ml}$ of concentrated sulfuric acid was added. In the case of the Amberchrome resin, the reaction was stopped after 90 seconds by adding an excess of ice water. The reaction was allowed to proceed for 6 minutes in the case of the Sarasep resin. The final product was then rinsed with methanol, water, and acetone. The cation exchange capacity was then determined by first passing $5 \mathrm{ml}$ of $1 \mathrm{M} \mathrm{HCl}$ through a known mass of resin to assure the resin was all in the protonated form. The resin was then rinsed with deionized water and tested with litmus paper to assure that any excess acid was washed from the reservoir. Then $5 \mathrm{ml}$ of a standardized $\mathrm{NaOH}$ solution was passed through the column. This was followed by another rinse with deionized water. The $\mathrm{NaOH}$ solution and water rinse was collected and titrated with a standard $\mathrm{HCl}$ solution. The capacity of the sulfonated Amberchrome resin was determined to be approx. $1.1 \mathrm{meq} / \mathrm{g}$, while the Sarasep resin was approx. $0.6 \mathrm{meq} / \mathrm{g}$.

The apparatus used for the solid-phase extraction (SPE) is shown in Figure 6 in the previous section. The SPE columns were obtained from Varian (Harbor City, CA, USA). 
Approximately $100 \mathrm{mg}$ of the resin was packed into the $55 \mathrm{x}$ $6.5 \mathrm{~mm}$ I.D. columns. The resin was held in place above and below by $20 \mu \mathrm{m}$ polyethylene frits. The resin bed height was approximately $12-15 \mathrm{~mm}$. The columns were connected to the laboratory-made reservoir by an adapter (Alltech Associates, Deerfield, IIl, USA). The flow rate was controlled by the air pressure applied to the reservoir.

The concentrated samples collected were analyzed using an HP $5880 \mathrm{~A}$ gas chromatograph with a flame ionization detector, an HP 5880 A Series Level 4 integrator, and an HP 7637A automatic sample injector (Hewlett-Packard, Avondale, PA, USA). The capillary column used was a supelco (Bellfonte, PA, USA) SPB-1.

\section{Procedure for $8 \mathrm{PE}$}

Prior to initial use, the sulfonated columns were cleaned by passing about $2 \mathrm{ml}$ of methanol and acetonitrile through them. This was followed by approximately $5 \mathrm{ml}$ of a 2 $M$ solution of $\mathrm{HCl}$ in methanol in order to protonate the sulfonic acid groups on the resin. The columns were then "wetted" with approximately $1 \mathrm{~mL}$ of methanol. The resin was not allowed to dry before passing the sample solution through the column.

The sample solutions were then prepared by adding a dilute methanol solution of several basic and neutral 
cor pounds to $10 \mathrm{ml}$ of deionized water so that the final concentration of each compound was $5 \mathrm{ppm}$. In the baseneutral group separation, the $\mathrm{pH}$ of this solution was then lowered to $\approx 2$ with $\mathrm{HCl}$. In the separation of strong bases from weak bases and neutrals a 0.1 buffer solution of sodium phosphate was used to adjust the $\mathrm{pH}$ to 7 . The sample was then added to the reservoir and the air pressure adjusted to give a sample flow rate of approximately $1 \mathrm{ml} / \mathrm{min}$. The column and reservoir were then rinsed with about $5 \mathrm{~mL}$ of water and air dried for several seconds.

The column was then rinsed with $1 \mathrm{ml}$ of methylene chloride to elute the neutral fraction. This was collected in a GC vial. An internal standard of $0.1 \mathrm{ml}$ of $500 \mathrm{ppm}$ quinoxaline in methanol was then added to the vial. The vial was capped, mixed with an orbital stirrer, and then analyzed by GC. A 1 Hl aliquot was injected with a split ratio of 1:40. Helium carrier gas was used at a flow rate of 15 $\mathrm{ml} / \mathrm{min}$. The oven temperature was held initially at $65^{\circ} \mathrm{C}$ for two minutes, then ramped at $15^{\circ} \mathrm{C} / \mathrm{min}$. to a final temperature of $225^{\circ} \mathrm{C}$. The basic fraction was then eluted with $1 \mathrm{ml}$ of either $2 \mathrm{M}$ methylamine or $2 \mathrm{MNH}_{3}$ in methanol. Quinoxaline was also added as an internal standard as before. The vial was then capped, mixed, and analyzed by GC under the 
previously given conditions. Recoveries were calculated by comparing the relative peak heights of collected samples to those not subjected to SPE. All results are an average of multiple trials ( $n \geq 3$ ). 


\section{RE8ULTB AND DIBCO8BION}

\section{Group Beparation of Neutral and Basic Compounds}

Previous work has shown that polystyrene divinyl benzene (PS/DVB) beads of high surface area (typically $\approx 400$ to 750 $\mathrm{m}^{2} / \mathrm{g}$ ) are very efficient for SPE of low concentrations of organic solutes in aqueous samples $(5,6)$. These resin beads still retain organic solutes when the beads are sulfonated provided that the degree of sulfonation is rather low. The sulfonated beads are able to retain protonated amine cations by an ion exchange mechanism. So long as the amines are present as cations, they are not washed off the resin by an organic solvent.

An intermediate capacity which facilitates adsorption by both ion exchange and hydrophobic interactions was sought. An optimum capacity of 0.6 to $1.0 \mathrm{meq} / \mathrm{g}$ was found. Lower capacities not only limited the amount of bases that could be adsorbed, but also showed less retention of neutrals. This could be largely due to the added hydrophilicity the exchange sites offered, causing more interaction between resin surface and solution. Larger capacities gave poorer retention of neutrals due to the reduction of underivatized surface area. The extent of sulfonation, as indicated by the exchange capacity of the sulfonated resin, can be kept low by sulfonation at $0^{\circ} \mathrm{C}$ for only a short period of time. 
The scheme for preconcentration of small amounts of organic solutes and their separation into groups was as follows. The sample was adjusted to an acidic $\mathrm{pH}$ with hydrochloric acid. This converted the basic compounds to the protonated cations. Then the sample was passed through a small column containing sulfonated resin in the $\mathrm{H}^{+}$form at a flow rate of approximately $1 \mathrm{ml} / \mathrm{min}$. The neutral organic solutes were eluted as a group with a small volume of methylene chloride. Then the basic fraction was eluted with a small volume of methylamine or ammonia in methanol.

Table IV shows excellent recovery of neutral organic solutes in water at $5 \mathrm{ppm}$. The gas chromatogram obtained is shown in Figure 12. In each case essentially complete elution is obtained with methylene chloride. The recoveries of some basic organic solutes studied are summarized in Table v. None of these compounds was eluted with methylene chloride except 2,3-dimethyl quinoxaline which is an extremely weak base and therefore behaves almost like a neutral compound. Some of the basic compounds were eluted well with methanol containing aqueous ammonia (see Figure 13), but stronger bases like hexylamine and cyclohexylamine were not eluted. However, as shown in Figure 14, methylamine in methanol eluted all of the bases efficiently. 
61

Table IV. Percent recoveries of neutral organics on sulfonated resin.

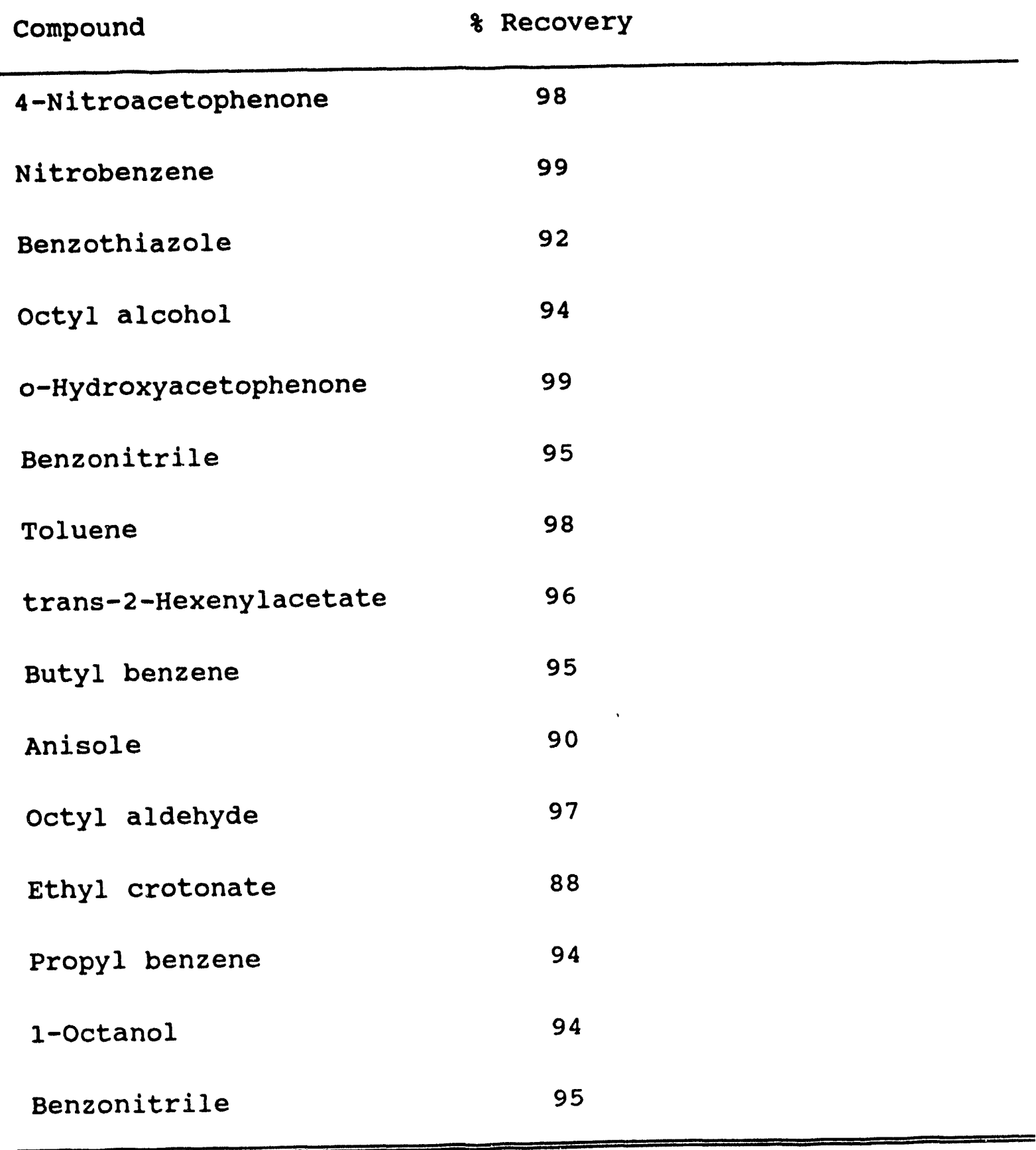




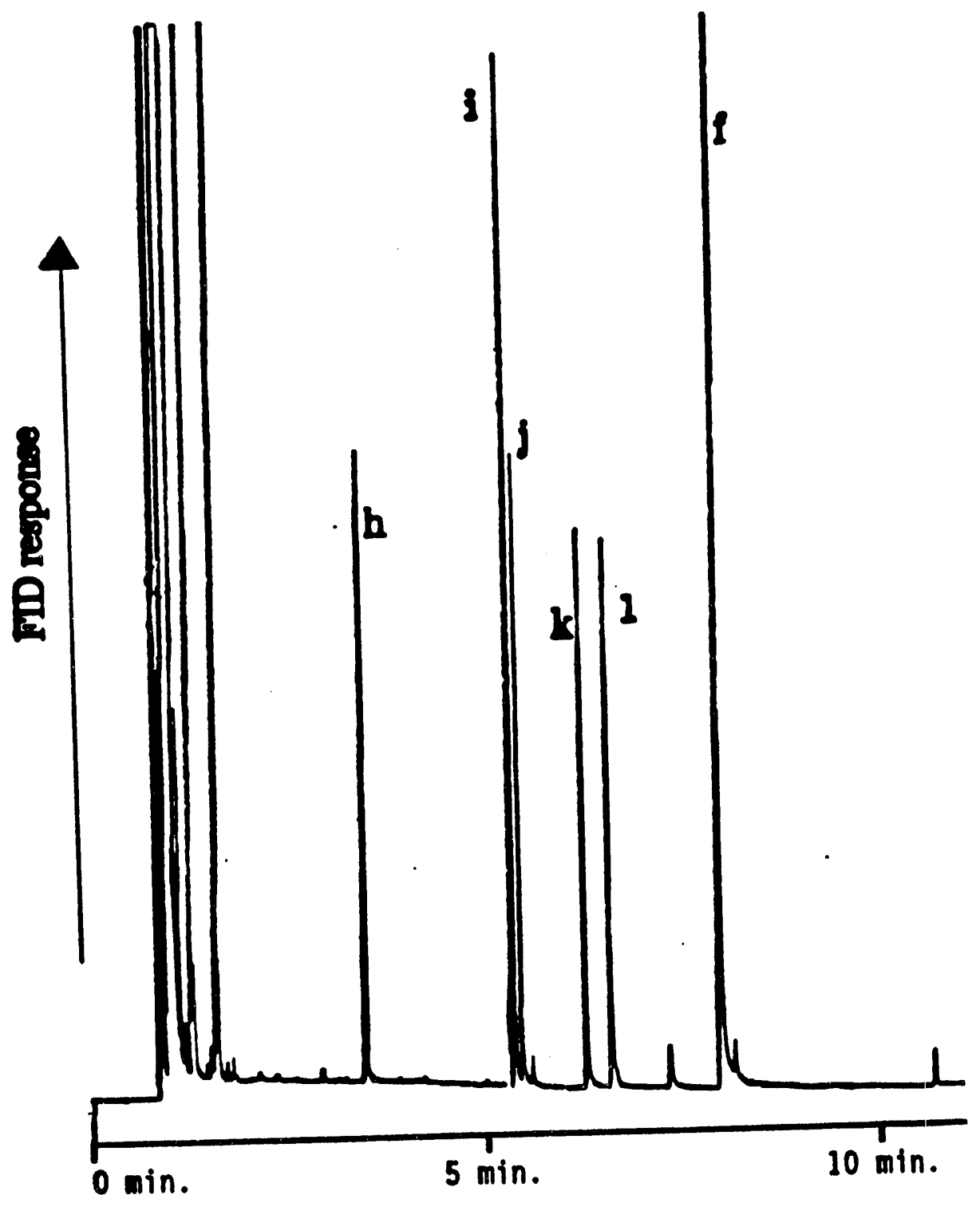

Figure 12. Gas chromatogram of neutral fraction concentrated on sulfonated resin and eluted with methylene chloride. (h=ethyl crotonate, $i=$ propyl benzene,

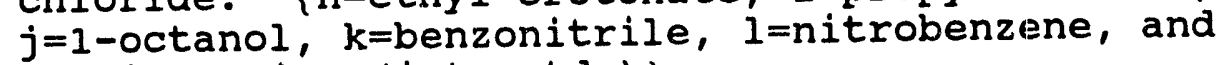
$f=q u i n o x a l i n e ~(i n t . ~ s t d$.$) ).$ 
63

Table V. Percent recoveries of bases concentrated on sulfonated resin.

compound

\& Recovery

$\left(\mathrm{NH}_{3}\right)$
\& Recovery

$\left(\mathrm{MeNH}_{2}\right)$

\begin{tabular}{lcc}
\hline Pyridine & 91 & 91 \\
Aniline & 95 & 99 \\
N,N'-Dimethyl aniline & 61 & 92 \\
Quinoline & 92 & 96 \\
Butyl amine & 0 & 103 \\
Octyl amine & 0 & 97 \\
Quinaldine & 92 & 95 \\
$2,3-D i m e t h y l$ quinoxaline & 63 & 63 \\
Hexyl amine & 0 & 95 \\
Cyclohexyl amine & - & 98 \\
N-Methyl aniline & - & 92 \\
sym-Collidin & - & 95 \\
Phenethyl amine & - & 98 \\
Ethyl pyridine & - & 93 \\
Isopropyl pyridine & - & 95 \\
$2,4-L u t i d i n e$ & - & 95 \\
\hline
\end{tabular}


64

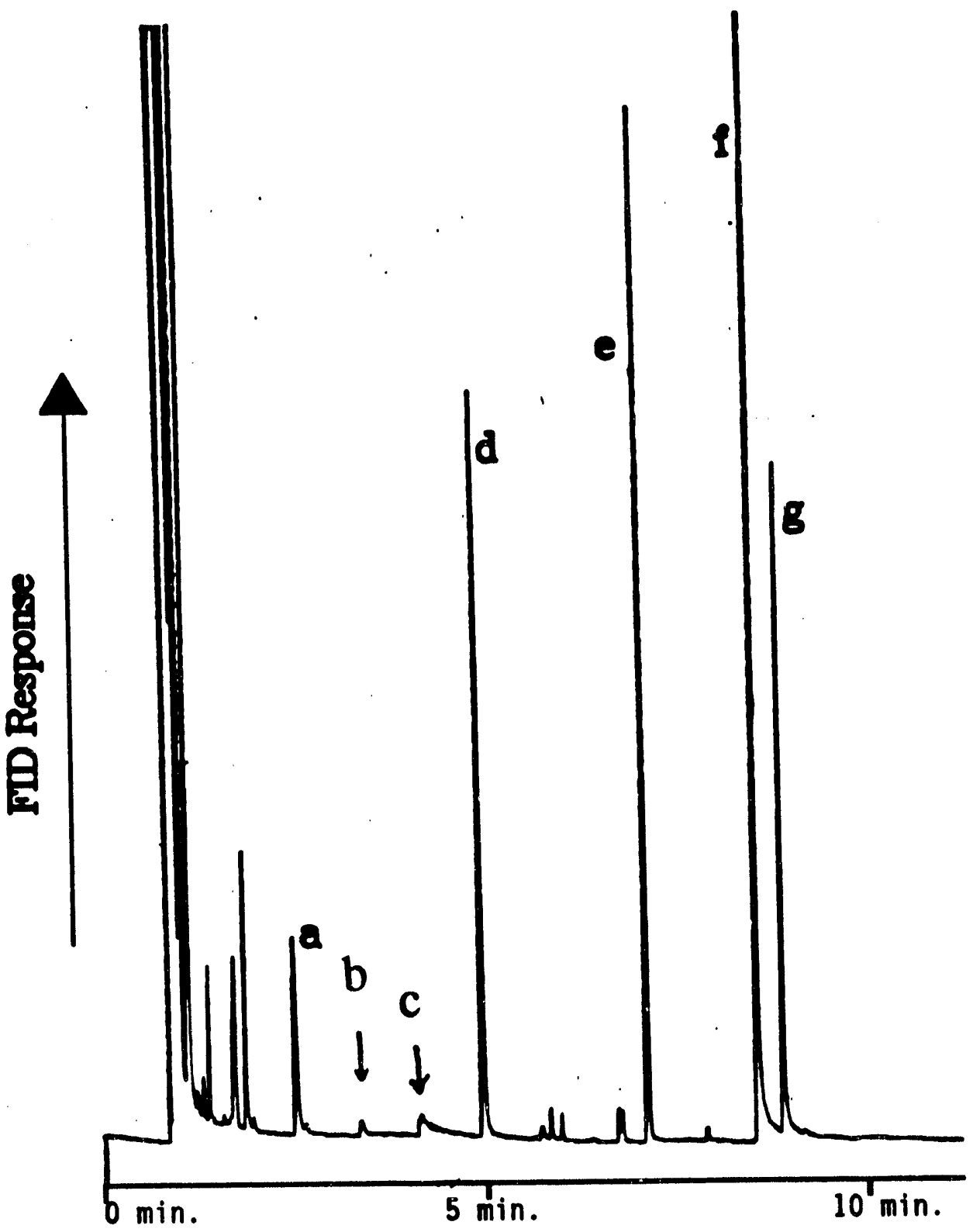

Figure 13. Gas chromatogram of basic fraction concentrated on cation exchange resin and eluted with ammonia in methanol. $\{a=$ pyridine, $b=h e x y l$ amine, $c=c y c l o h e x y l$ amine, $d=2,4-$ lutidine, $e=N, N^{\prime}$-dimethyl aniline, $f=q u i n o x a l i n e$ (int. std.), g=quinoline . Note absence of peaks for strong bases. 


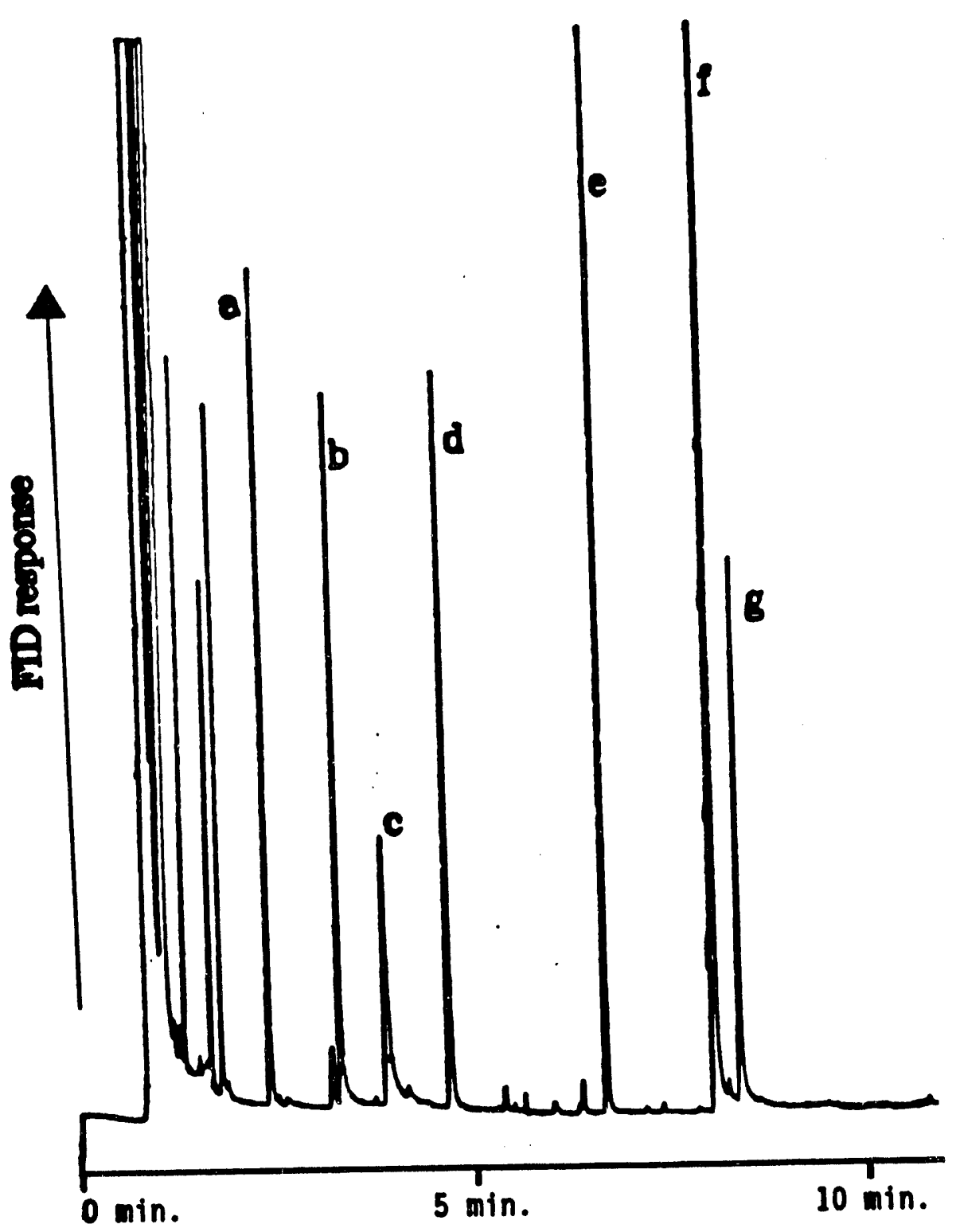

Figure 14. Gas chromatogram of basic fraction concentrated on cation exchange resin and eluted with methylamine in methanol. (a=pyridine, $b=h e x y l$ amine, $c=$ cyclohexyl amine, $d=2,4$-lutidine, $e=N, N^{\prime}-$

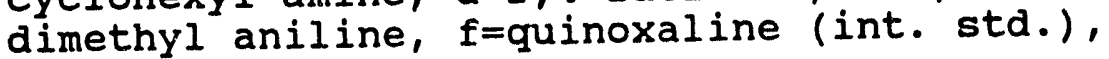
$g=q u i n o l i n e\}$. 
Methylamine $\left(\mathrm{pK}_{\mathrm{b}}\right.$ in water $\left.=3.1\right)$ is a stronger base than ammonia $\left(\mathrm{pK}_{\mathrm{b}}=4.8\right)$. In the $\mathrm{GC}$ step methylamine is quite volatile and elutes well before any of the sample solutes. This property of ammonia allows a three-step elution process that further fractionates the bases. First, methylene chloride is added to elute neutrals. The second elution is then ammonia in methanol or acetonitrile. In this step the weaker bases $\left(\mathrm{pK}_{\mathrm{b}}>5\right)$ such as quinoline or quinaldine are eluted. Then in the final elution, methylamine in acetonitrile is used as the eluent. This will then elute the remaining stronger amines.

The effect of $\mathrm{pH}$ on the group separation was investigated. Results were compared for samples buffered at pH 2.0 with $0.1 \mathrm{M}$ sodium dihydrogen phosphate and $\mathrm{pH} 7.0$ using disodium hydrogen phosphate. The results in Table VI show that at pH 2.0 neutral compounds were eluted with methylene chloride and basic compounds with methylamine in methanol, as expected. Recoveries averaged lower with the $\mathrm{pH}$ 2.0 phosphate buffer than when the sample was acidified with $\mathrm{HCl}$. At $\mathrm{pH} 7.0$ the weaker bases (aniline, quinoline, etc.) were not protonated, and therefore were eluted with the neutral group. The stronger bases (hexylamine, octylamine, etc.) remained protonated at this $\mathrm{pH}$, and therefore were not eluted until the methylamine in methanol wash step. Thus an 
Table VI. Recovery from $10 \mathrm{ml}$ aqueous solution buffered at $\mathrm{pH} 2.0$ and 7.0 with $0.1 \mathrm{M}$ phosphate. All samples 5 ppm. Capacity of cation exchange resin is $0.89 \mathrm{meq} / \mathrm{g}$.

\begin{tabular}{|c|c|c|c|c|}
\hline Compound & $\mathrm{CH}_{2} \mathrm{Cl}_{2}$ & $\begin{array}{c}\mathrm{MeNH}_{2} \\
(\mathrm{pH}=2) \\
\end{array}$ & $\begin{array}{l}\mathrm{CH}_{2} \mathrm{Cl} \\
2 \\
\end{array}$ & $\begin{array}{c}\mathrm{MeNH}_{2} \\
(\mathrm{pH}=7)\end{array}$ \\
\hline pyridine & 0 & 44 & 43 & 0 \\
\hline toluene & 95 & 0 & 101 & 0 \\
\hline cyclohexyl amine & 0 & 76 & 0 & 76 \\
\hline aniline & 0 & 88 & 91 & 0 \\
\hline benzyl alcohol & 93 & 0 & 98 & 0 \\
\hline benzonitrile & 98 & 0 & 90 & 0 \\
\hline octyl alcohol & 93 & 0 & 99 & 0 \\
\hline hexyl amine & 0 & 91 & 0 & 90 \\
\hline octyl amine & 0 & 91 & 0 & 90 \\
\hline p-ethyl phenol & 92 & 0 & 91 & 0 \\
\hline o-hydroxy acetophenone & 95 & 0 & 94 & 0 \\
\hline benzothiazole & 94 & 0 & 100 & 0 \\
\hline quinoline & 0 & 90 & 93 & 0 \\
\hline quinaldine & 0 & 90 & 88 & 0 \\
\hline 2,3-dimethyl quinoxaline & 85 & 0 & 94 & 0 \\
\hline 4-nitroacetophenone & 75 & 0 & 86 & 0 \\
\hline
\end{tabular}


additional group separation of aliphatic amines from aromatic amines and heterocyclic nitrogen compounds is possible.

This type of group separation within a group provides an additional mode of separation not previously demonstrated. It can be done by either of two methods, $\mathrm{pH}$ control in the loading step, or using a base of appropriate strength in the elution step.

\section{Group separation of Bases and Neutrals in Non-polar solution}

Volatile organic bases contained in a nonpolar solvent could be extracted as a group using this membrane. An example of this is shown in Figure 15 with a toluene solution containing trace amounts of piperidine, 3-picoline, and 2ethyl pyridine. These early eluting bases cannot be separated from the large solvent peak by ordinary gas chromatographic methods. By adding sufficient $\mathrm{HCl}\left(10^{-4} \mathrm{M}\right)$, the bases are protonated and thus retained by the sulfonic acid groups on the resin imbedded in the membrane. This acid concentration is an optimum and crucial to the success of the extraction. A higher concentration results in increased competition for the cation exchange sites, while a lower concentration appears to give incomplete protonation. Both of these scenarios lead to decreased extraction efficiency. After the toluene solution has passed through the membrane, the SPE column is blown dry. The bases are then eluted with 


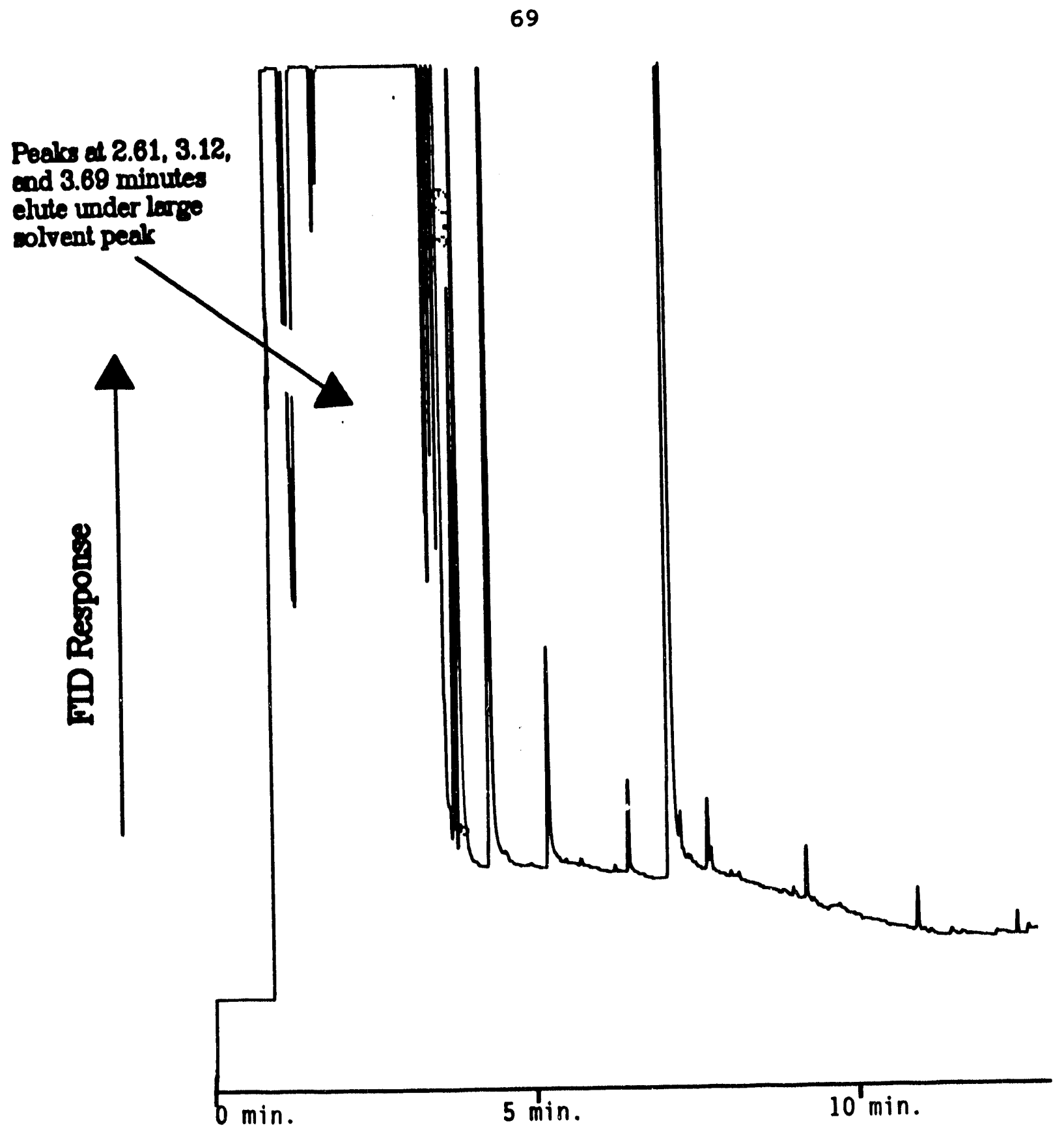

Figure 15. Gas Chromatogram of trace concentration (6 ppm) volatile bases in toluene (no extraction performed). 
2M methyl amine in acetonitrile and determined by gas chromatography. An example of the resultant chromatogram is shown in Figure 16. The average recoveries of 4 trials is shown in Table VII.

This technique is also useful in extracting bases present at trace concentrations from complex non-aqueous mixtures. An example of this is shown in Figure 17. Five bases, benzyl amine, phenethyl amine, tributyl amine, quinoiine, and quinaldine are present at $10 \mathrm{ppm}$ in a toluene solution containing bromobenzene, 3-phenyl-propanol, octanol, decanol, dodecanol, and tetradecanol all at $1500 \mathrm{ppm}$. Direct analysis of the solution for the bases present by GC is impossible due to the large peaks present due to the various neutrals. But if the basic fraction is extracted by $S P E$, analysis by GC is possible. Two milliliters of this solution was acidified with $\mathrm{HCl}(0.0001 \mathrm{M})$ and passed through the membrane at a flow rate of $1 \mathrm{ml} / \mathrm{min}$. The membrane was then allowed to blow dry with air pressure for a period of 1 minute. The bases were then eluted with $1 \mathrm{mI}$ of $2 \mathrm{M}$ methyl amine in acetonitrile to which $0.1 \mathrm{ml}$ of quinoxaline internal standard was added. Both the basic and neutral fractions were analyzed by GC. Excellent recoveries were obtained for the bases. The results are given in Tables VIII and IX. 
71

Table VII. Percent Recovery of Basic compounds when extracted from toluene onto cation exchange membrane and eluted with methyl amine.

Basic compound

$$
\text { Piperidine }
$$

3-Picoline

2-Ethyl pyridine
\& Recovery

$$
94.7
$$

100.2

96.5 


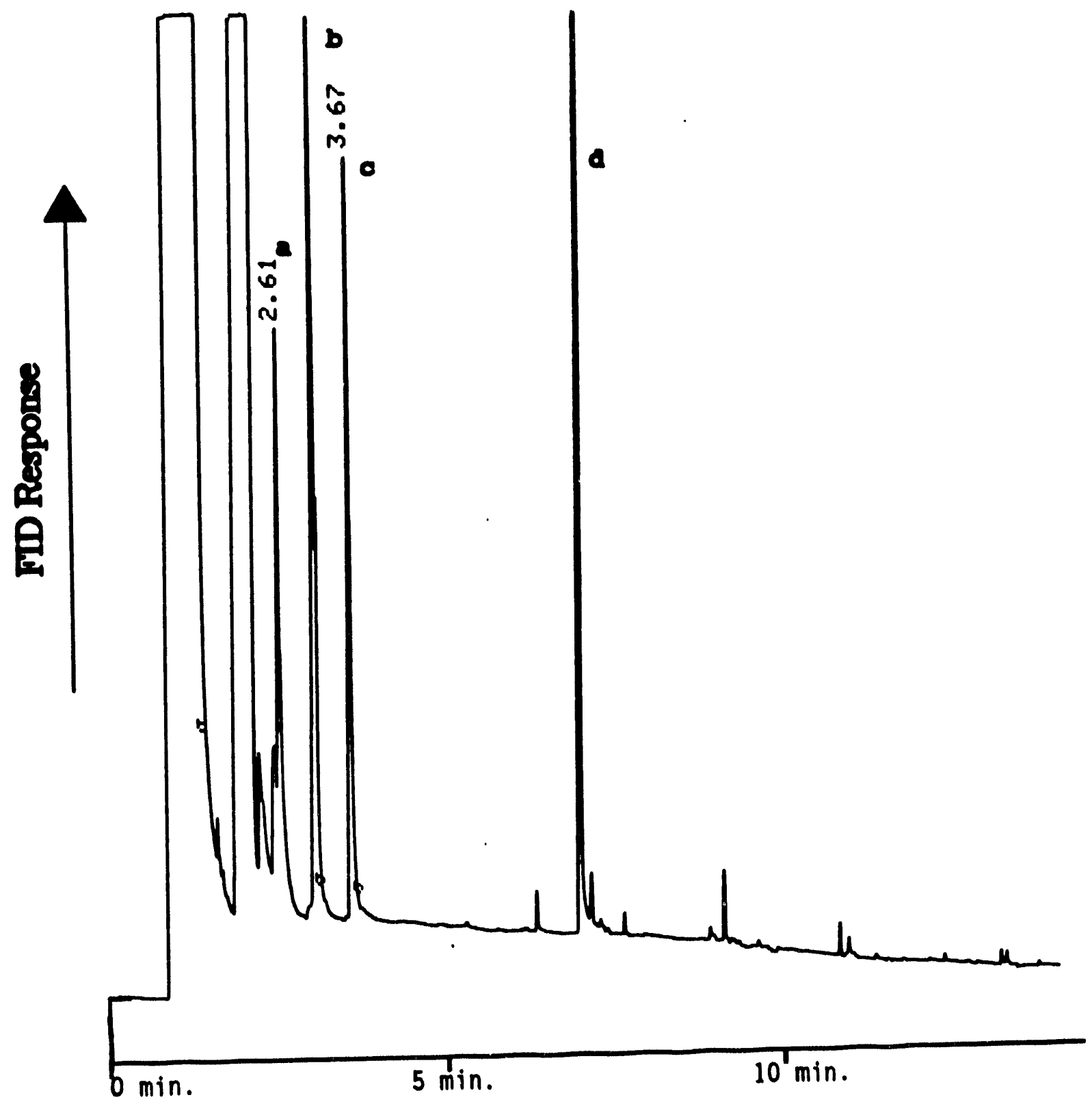

Figure 16. Gas chromatogram of early eluting bases after extraction from toluene. Bases are protonated by adding $\mathrm{HCl}$ to a mixture of $10^{-4} \mathrm{M}$, passed through the sulfonated membrane, blown dry, and eluted with $1 \mathrm{~mL}$ of $2 \mathrm{M}$ methyl amine in acetonirile. ( $a=$ piperidine, $b=3-p i c o l i n e, c=2$-ethyl pyridine, $d=$ quinoxaline (internal standard\}). 
73

Table VIII. Recoveries of neutral compounds from non-polar solution in base/neutral fractionation using sulfonated membrane.

neutral organic compounds

\& recovery

octane

benzonitrile

nitrobenzene

isophorone

o-hydroxy propiophenone

butyl benzene

3-phenyl-1-propanol

phenetole
97

104

98

103

97

96

100

105 
Table IX. Recoveries of bases extracted from non-polar solution with cation exchange membrane.

basic organic compounds

benzyl amine

tributyl amine

phenethyl amine

quinoline

quinaldine

3-picoline

2-ethyl pyridine

isopropyl pyridine

$\mathrm{N}, \mathrm{N}^{\prime}$-dimethyl pyridine
\& recovery

94

95

99

99

98

100

102

95

99 
Examples of the chromatograms obtained are shown in Figures 17 and 18 .

Ion Exchange separation of Organic Bases in Non-aqueous Media

In an effort to better understand the forces present in ion exchange process in the uptake of organic bases by cation exchange resin, we studied the ion-exchange separation of organic bases in non-aqueous eluant. When an aqueous eluant is used in these processes, hydrophobic interactions play a significant role in the processes. We attempted to minimize the role of these hydrophobic effects by performing a chromatographic separation in a $100 \%$ alcohol eluant.

Very nice separations were achieved by this method. Figure 19 shows the separation of aniline, $N^{\prime}-$ methyl aniline, and $N_{,} N^{\prime}$-dimethyl aniline on just a $5 \mathrm{~cm}$ sulfonated column in a butanol/methanol eluant. By varying the chain length of the alcohol, we could control the relative polarity of the eluant. Table $\mathrm{X}$ shows the retention times of several bases in various alcohol mixtures. Looking at the smaller, more polar bases like pyridine and aniline, it is apparent from the retention times shown that in these eluants the separation is purely one of ion-exchange. The addition of a longer chain alcohol seems to play little role in the retention of the base when compared to the retention of these bases in methanol alone. Looking at larger bases, such as 


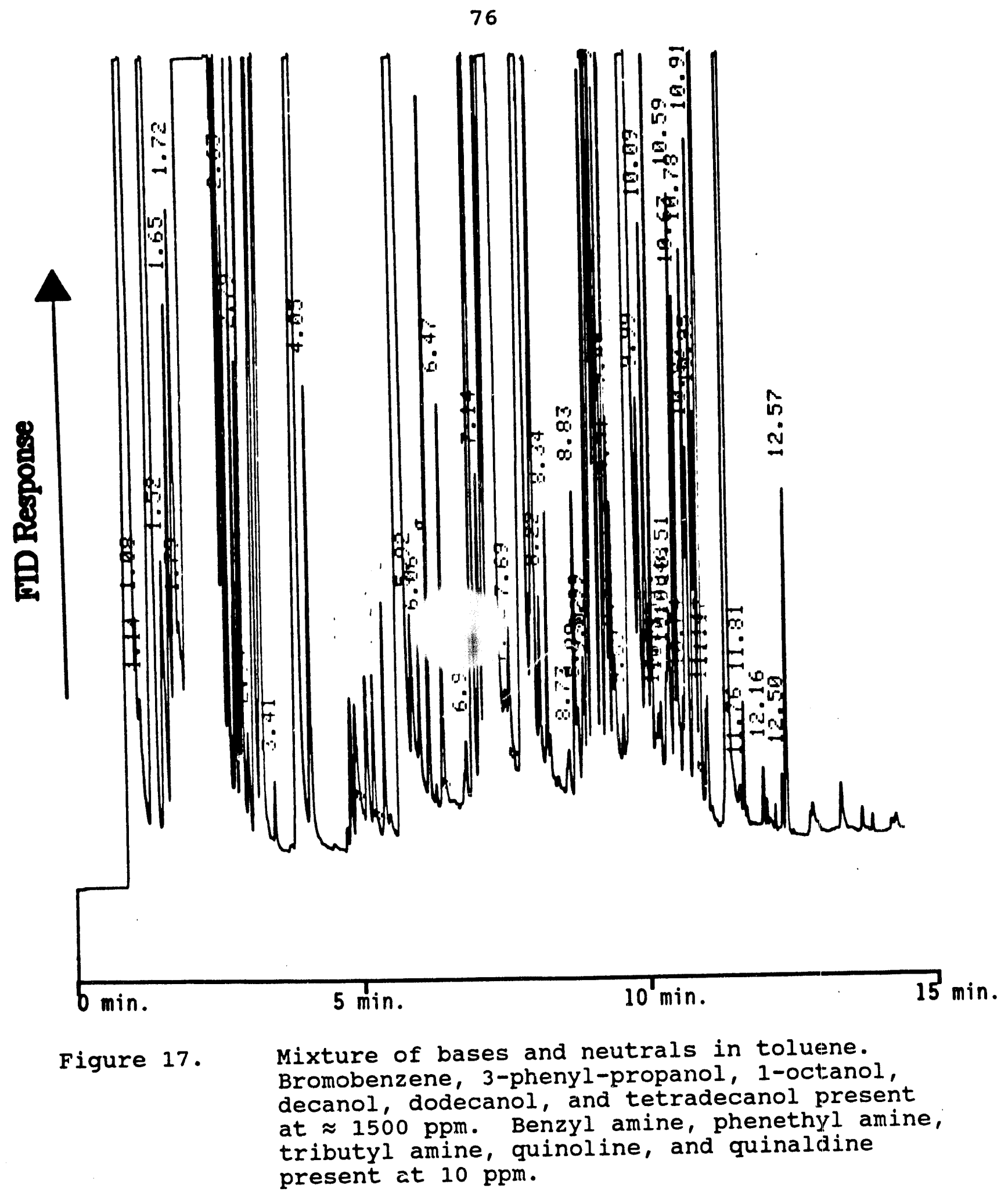




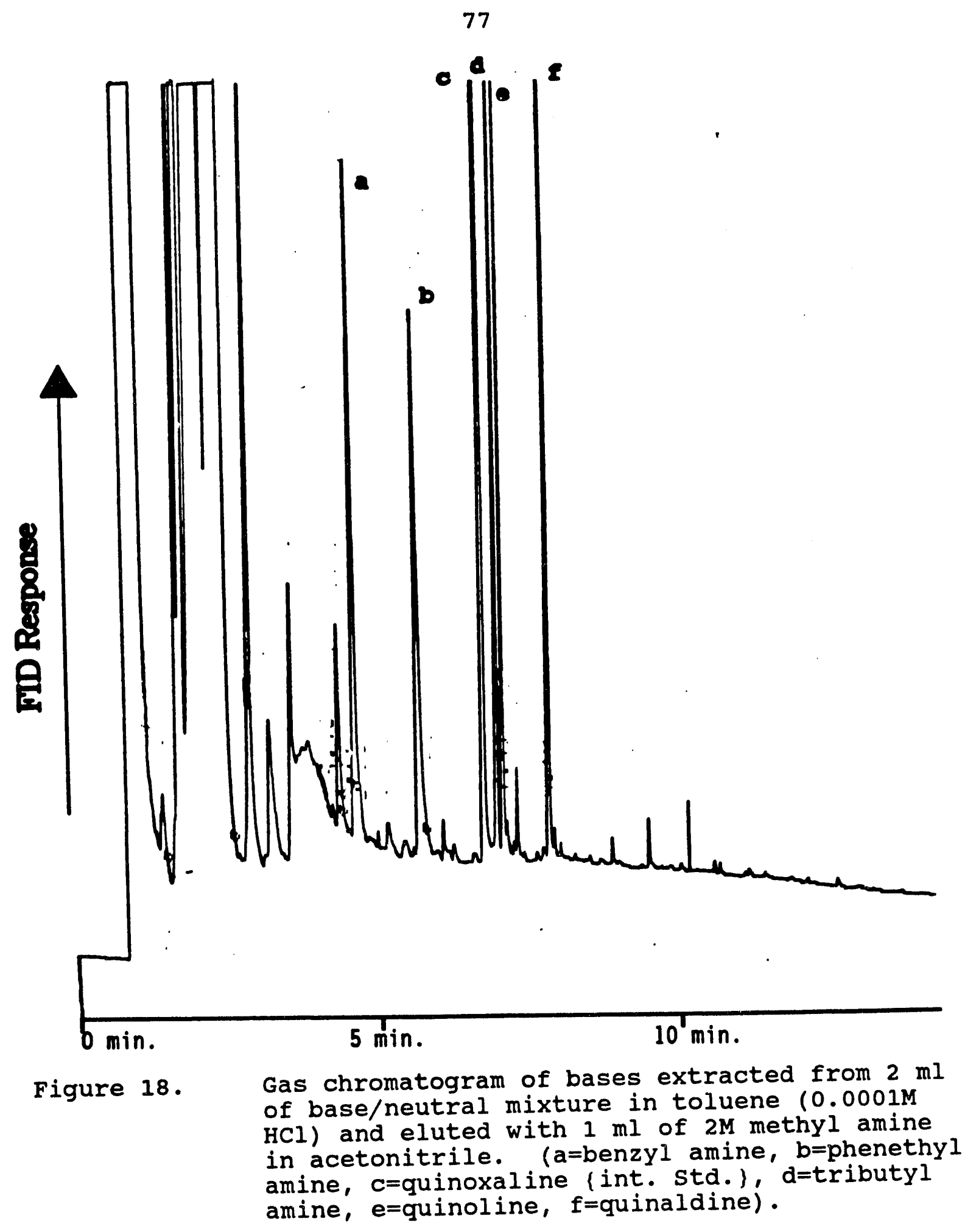




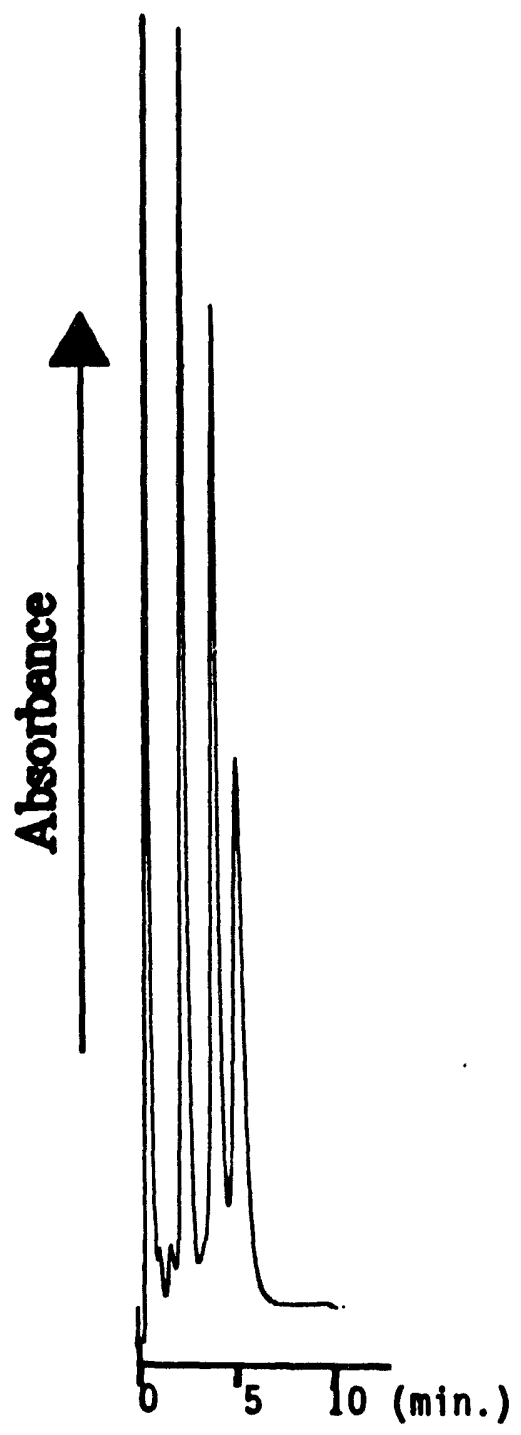

Figure 19. Chromatographic separation of aniline, $\mathrm{N}$-methyl aniline, and $\mathrm{N}, \mathrm{N}^{\prime}$-dimethyl aniline in 35/65\% butanol/methanol eluant on $5 \mathrm{~cm}$ sulfonated column $(0.15 \mathrm{meq} / \mathrm{g})$. Flow rate $=1 \mathrm{ml} / \mathrm{min}$. 
Table $x$. Retention times of bases in non-aqueous solutions acidified with $0.02 \mathrm{M}$ methanesulfonic acid.

\begin{tabular}{lcccc} 
& & & $(65 \% \mathrm{MeOH} /$ & $(65 \% \mathrm{MeOH}$ \\
organic base & $(\mathrm{MeOH})$ & $(65 \% \mathrm{MeOH} / 35 \% \mathrm{EtOH})$ & $35 \%$ PropOH $)$ & $/ 35 \% \mathrm{ButOH})$ \\
\hline pyridine & 3.76 & 3.87 & 3.82 & 3.78 \\
$\begin{array}{l}\text { quinoline } \\
\text { 5,6-benzoquinoline }\end{array}$ & 13.74 & 5.79 & 5.63 & 5.32 \\
aniline & 1.91 & 10.21 & 9.93 & 8.67 \\
$\mathrm{~N}$-methyl aniline & 3.12 & 2.18 & 1.92 & 1.91 \\
$\mathrm{~N}, \mathrm{~N}$-dimethyl aniline & 6.12 & 3.94 & 3.55 & 3.50 \\
\hline \hline
\end{tabular}


80

$N, N^{\prime}$-dimethyl aniline and 5,6-benzoquinoline, we see that the retention of these bases is reduced as the chain length of the alcohol increases. This indicates that hydrophobic interactions play a larger role in the separation of these bases. In fact, it most likely these interactions that control the actual separation of the bases. As these interactions are reduced, the retention times of similar bases like quinoline and 5,6-benzoquinoline become closer. This should allow for the optimization of these separations. We also looked at the role of the acid in the separation. By varying the acid concentration of the eluent while keeping the composition of the eluent the same, we were able to plot $\log t_{r}$, vs. $\log$ [acid]. In theory, the slope of this plot should be -1 . Table XI shows that in most instances our results were very close to this value.

\section{On-line separation}

In $1991 \mathrm{~J}$. Sun and J. S. Fritz (38) demonstrated a method for the on-line extraction of basic compounds by placing a sulfonated polymeric resin in the injection port of a gas chromatograph. This sulfonated resin, placed in the injection liner and heid in place with glass wool, extracted basic compounds from a sample solution while letting neutrals pass. 
81

Table XI. Slopes of plots of $\log t_{r}^{\prime}$ vs. $\log$ [acid]

\begin{tabular}{lccc} 
organic base & $\begin{array}{c}\text { slope } \\
(\mathrm{MeOH})\end{array}$ & $\begin{array}{c}\text { slope } \\
(65 \% \mathrm{MeOH} / 35 \% \mathrm{EtOH})\end{array}$ & $\begin{array}{c}\text { slope } \\
(65 \% \mathrm{MeOH} / 35 \% \mathrm{ButOH})\end{array}$ \\
\hline pyridine & -1.10 & -1.00 & -1.05 \\
quinoline & -0.984 & -0.988 & -1.02 \\
S,6-benzoquinoline & -1.00 & -0.982 & -1.06 \\
aniline & -1.00 & -0.982 & -0.977 \\
$\mathrm{~N}$-methyl aniline & -1.02 & -1.02 & -1.04 \\
$\mathrm{~N}, \mathrm{~N}$-dimethyl aniline & -1.02 & -1.02 & -1.08 \\
\hline \hline
\end{tabular}


82

The abstraction of basic compounds was essentially quantitative, but as yet no method has been developed for the elution of the adsorbed bases.

Simple modifications of this procedure allow for the group separation of bases and neutrals to be done on-line. A small $5 \mathrm{~cm}$ column was packed with sulfonated resin of a capacity of $0.15 \mathrm{meg} / \mathrm{g}$. An ion exchange separation of organic bases can be performed on this column in a nonaqueous solution containing acid. In a non-aqueous solution, retention of the bases and neutrals by hydrophobic interaction can be minimized. In this case then, neutrals will be unretained and pass through the column in the void volume.

An example of this separation is shown with a mixture containing three neutrals and three bases in acetonitrile. This group separation is shown in Figure 20. A separation of these bases was performed in an eluent containing $65 \%$ methanol and 35\% butanol acidified with $0.02 \mathrm{M}$ methanesulfonic acid. The mixture containing the neutrals and bases was injected onto the column. The first $\mathrm{mL}$ of eluant coming off the column was collected in a GC vial, an internal standard was added, and the eluant was then analyzed by a gas chromatograph. 


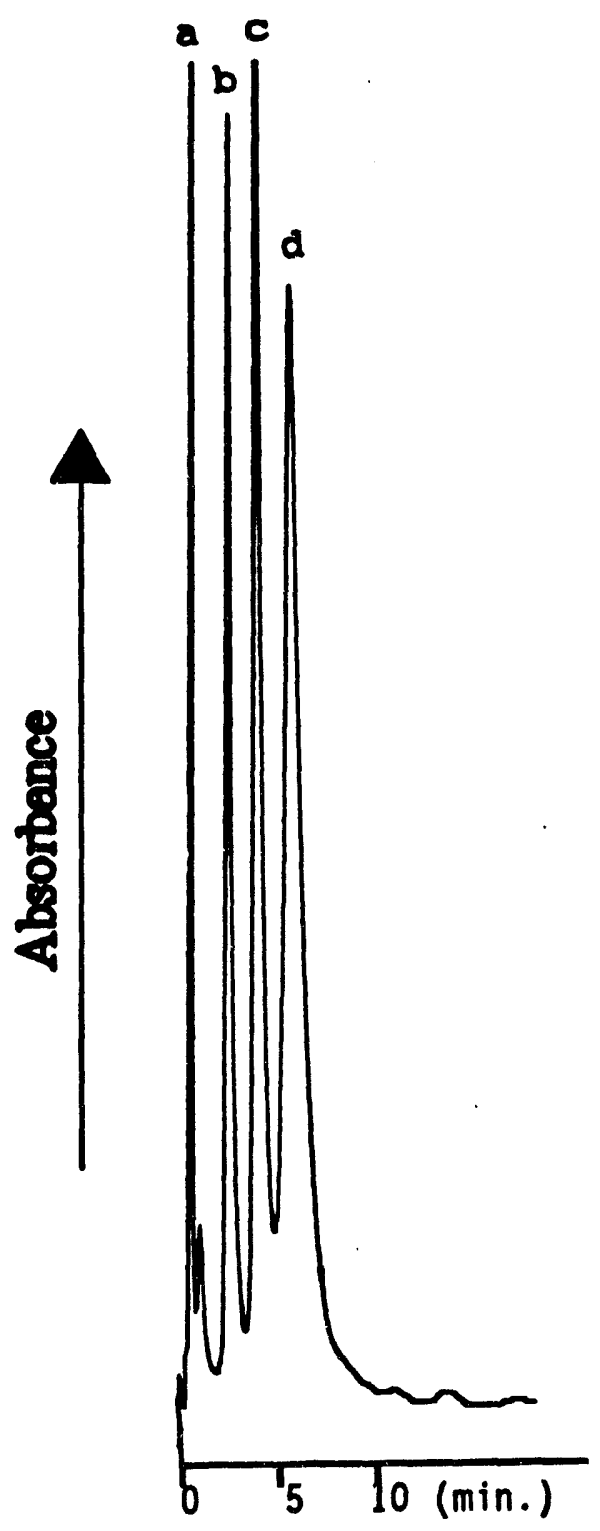

Figure 20. Chromatographic group separation of neutrals (a) and bases pyridine (b), quinoline (c), and benzoquinoline (d) in 35/65\% butanol/methanol eluant on $5 \mathrm{~cm}$ sulfonated column $(0.15 \mathrm{meq} / \mathrm{g})$. Flow rate $=1 \mathrm{ml} / \mathrm{min}$. 


\section{4}

The gas chromatograph of these neutrals is shown in Figure 21. The recoveries of these neutrals were all very good, as shown in Table XII. This type of group separation could be easily automated and done with column switching. 


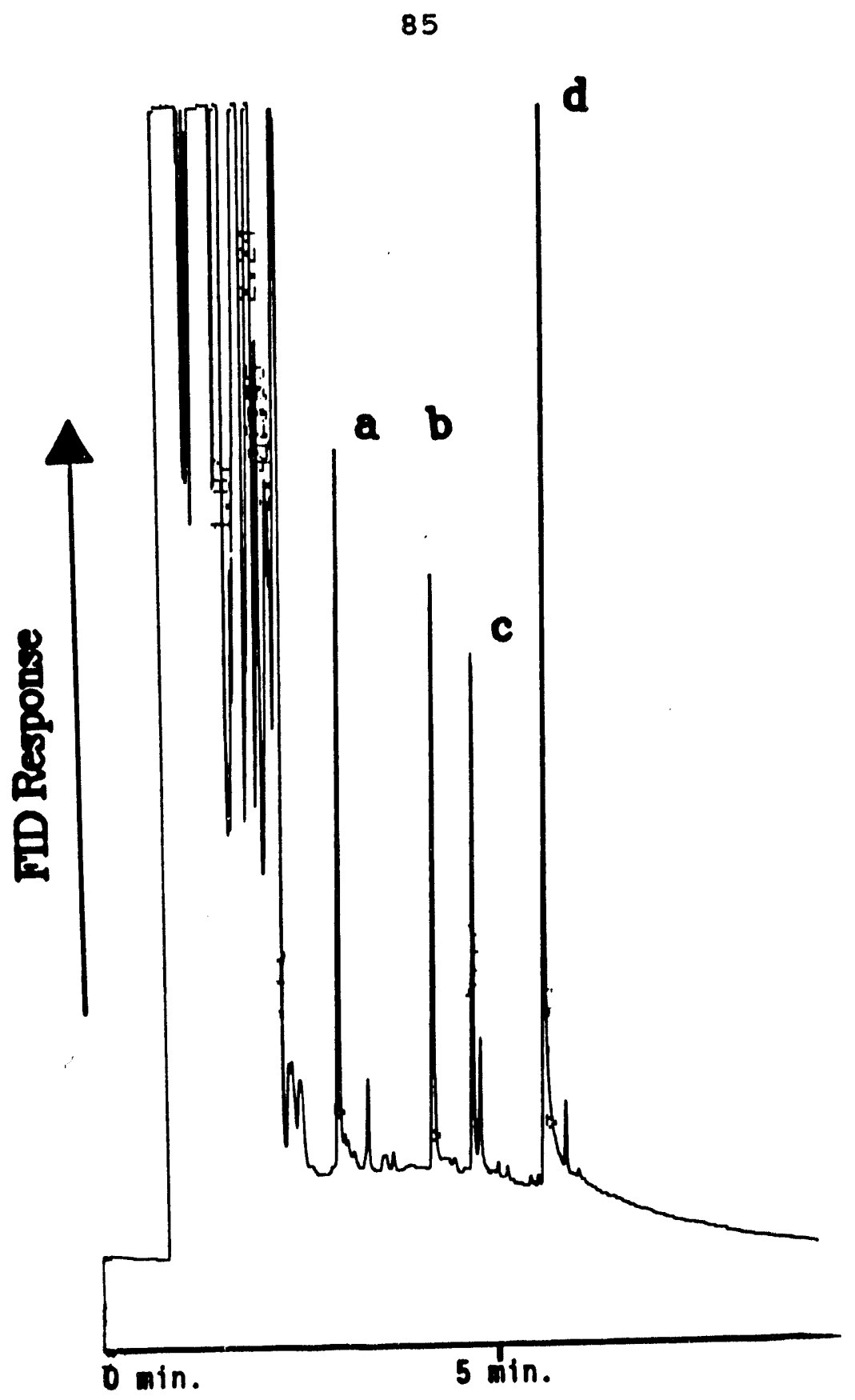
Figure 21. Gas chromatograph of neutral fraction collected in
void volume in on-line separation. (a=benzonitrile, $b=n i t r o b e n z e n e, c=i s o p h o r o n e, d=q u i n o x a l i n e$ (int. std. \}) 
Table XII. Percentage Recoveries of neutral compounds in LC void volume.

Neutral Compound

of Recovery
Octane

Benzonitrile

Nitrobenzene

Isophorone
98

100

90

100 


\section{CONCLUSION}

Fractionation of samples prior to chemical analysis is important for a number of reasons. It provides chemical information about analytes, simplifies complex samples, and provides an added separation mode to simplify analyses. In addition to a sample cleanup, it can allow for the identification and determination of trace components present in samples containing large amounts of other sample compounds.

The fractionation schemes demonstrated here have proven to be effective with a variety of neutral compounds and both strong and weak bases. Fractionation is achieved for samples contained in polar and nonpolar matrices. It appears to be quite predictable, fast, and efficient. The recoveries of sample components are quantitative and reproducible. 
RART III. ION EXCHANGE PRECONCENTRATION AND GROUP GEPARATION OF ACIDIC AND NEUTRAL COMPOUNDS 


\section{INTRODUCTION}

As in section II, we studied the use of partially derivatized ion exchange resins as a method of performing both preconcentration and group separation by SPE. Once again a medium capacity resin was used in a dual adsorption mode (see Figure 1). Neutral organic molecules in aqueous solution attach themselves hydrophobically at unreacted surface sites on the polymer, while anions will adhere electrostatically at the anion exchange sites.

The reaction used in the preparation of the resin was first described by Barron and Fritz in 1983 (41). The first step of this reaction is the chloromethylation step:

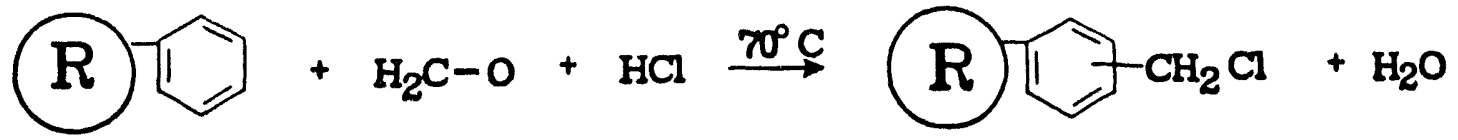

This is the step that is used to determine the capacity of the resin. This is done by controlling the reaction time at a constant temperature of $70^{\circ} \mathrm{C}$. The second step, amination, is allowed to react to completion:

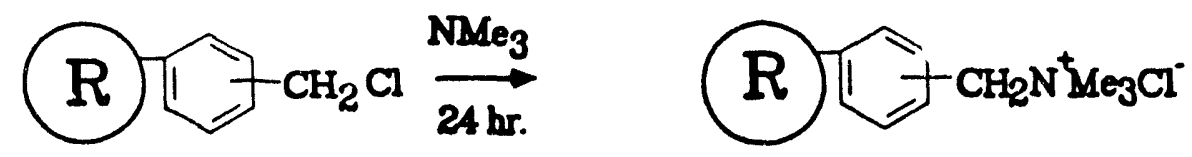




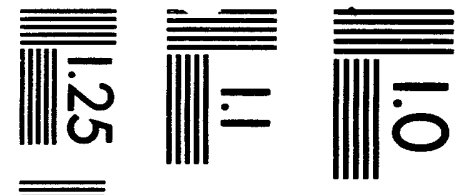

$$
\begin{aligned}
& \||||| \text { 六 }
\end{aligned}
$$

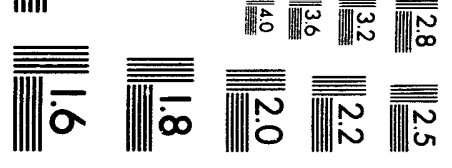



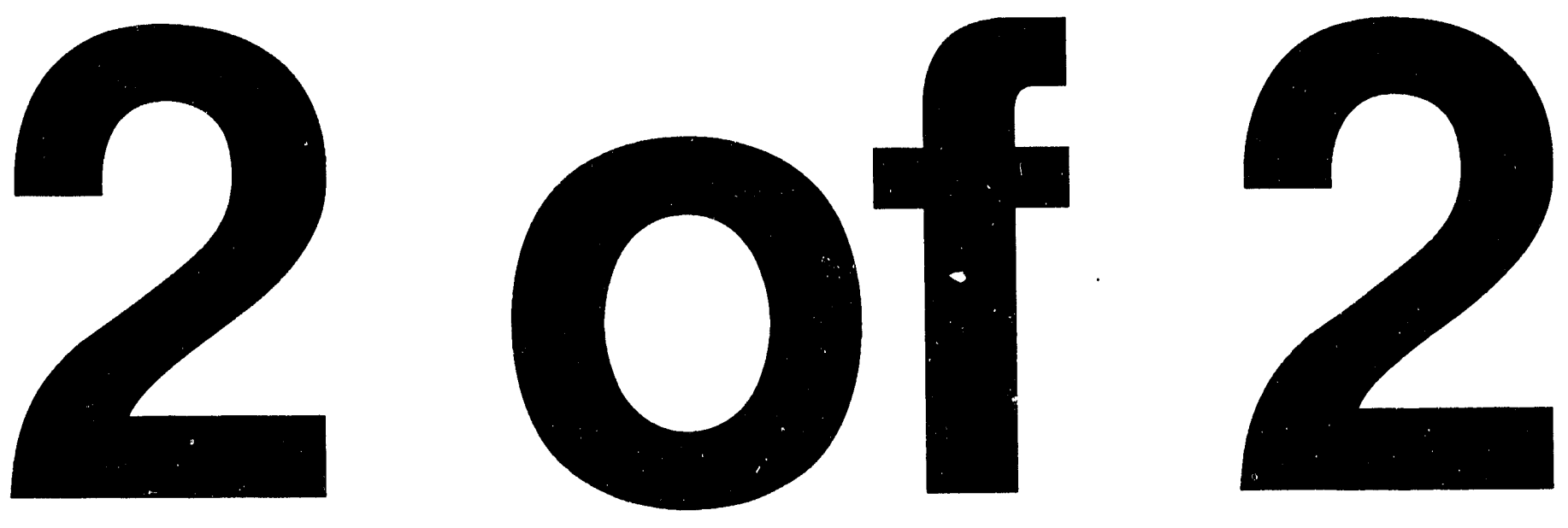


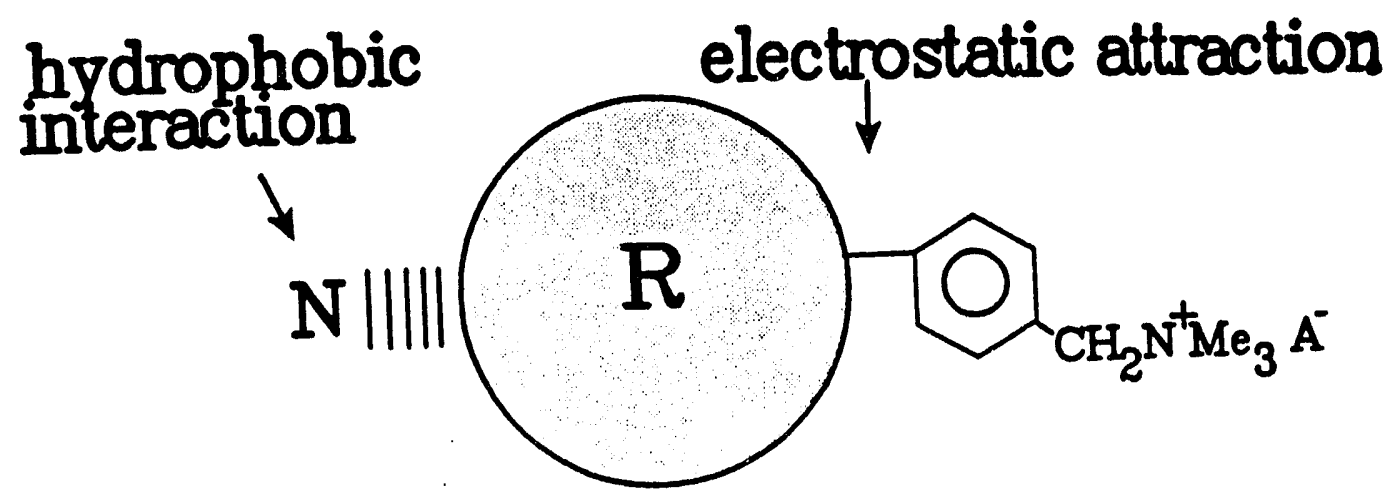

\footnotetext{
Figure 22. Diagram illustrating dual adsorption mode at single resin particle.
} 
91

Once the sample analytes have been adsorbed onto the resin particle, a two-step elution process is again used to separately elute the neutral and acidic fractions. The neutrals are eluted with $1 \mathrm{ml}$ of nonpolar solvent such as methylene chloride, and the acids are subsequently washed off the column with an acetonitrile or methanol solution containing an acid such as $\mathrm{HCl}$ or methanesulfonic acid. An outline for this scheme is shown in Figure 23. This resin has also been used for the abstraction of organic acids from nonpolar matrices. This type of extraction is often vital, as the chromatographic methods of determination differ for volatile organics and carboxylic acids. 

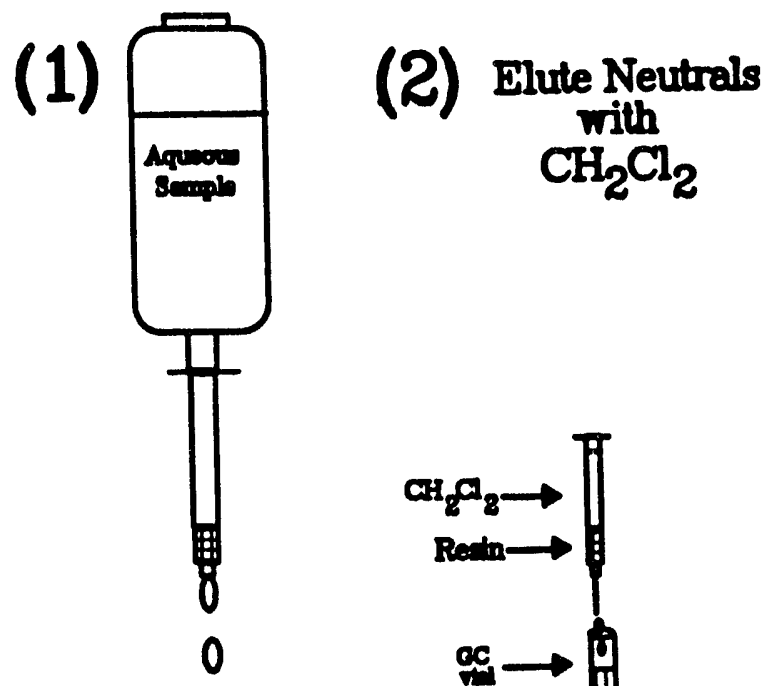

(3) Elute Carbosglic ecids
with $\mathrm{HCl}$ in Acetonitrile Elute Phenols with $\mathrm{HCI}$ in $\mathrm{MeOH}$
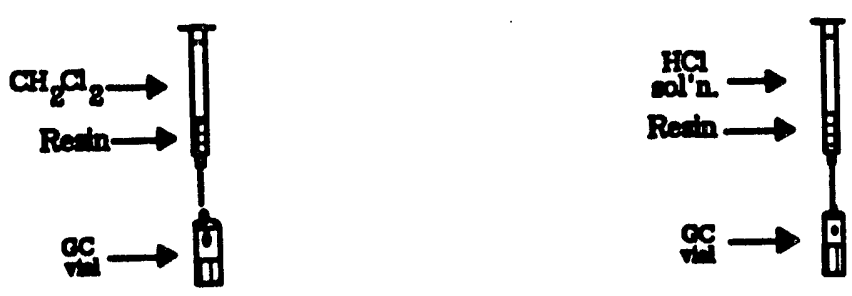
Figure 23. Concentration and two step elution scheme for group separation of neutral and acidic organic analytes.




\section{EXPERIMENTAL}

\section{Reagents and Chemicals}

The reagents used for the derivatisation of the ionexchange resins were of analytical grade. Reagents used and analytes studied in SPE were $>998$ pure and used as obtained from Aldrich, Fisher, and Kodak. Laboratory distilled water was further purified using a Barnstead Nanopure II system (Sybron Barnstead, Boston, MA, USA).

The extractions were done using two different resins: a highly porous $40 \mu \mathrm{m}$ cross-linked polystyrene resin, Amberchrome 161 (Supelco, Bellefonte, PA, USA), and an $8 \mu \mathrm{m}$ PS/DVB resin, SRP 80 (Sarasep, Inc., Santa Clara, CA, USA).

The two step procedure for the preparation of the anion exchange resin is as follows. In an oil bath at $70^{\circ}, 1 \mathrm{~g}$ of resin was wetted with $1 \mathrm{ml}$ of glacial acetic acid. To this $25 \mathrm{ml}$ of a $2.2 \mathrm{M}$ solution of paraformaldehyde in concentrated hydrochloric acid was added. This chloromethylation step was allowed to proceed with slight stirring for 24 (for the Amberchrome resin) to 48 (for the SRP resin) hours. The resin was then filtered and rinsed with water and methanol. The resin was then aminated by adding $25 \mathrm{ml}$ of $25 \%$ trimethylamine in ethanol and stirring at room temperaturee for 24 hours. The anion exchange product was then rinsed with methanol, water, and acetone. 
The anion exchange capacity was determined by titration. A known mass of resin was put in its basic form by passing 5 ml of $1 \mathrm{M} \mathrm{NaOH}$ over it. The resin was then rinsed with deionized water and tested with litmus paper to assure that excess $\mathrm{NaOH}$ was completely rinsed. Then, $5 \mathrm{ml}$ of a standard $\mathrm{HCl}$ solucion was passed through the resin. This was followed by another rinse with deionized water. The $\mathrm{HCl}$ and water rinse were collected and titrated with a standard $\mathrm{NaOH}$ solution. The capacity of the Amberchrome resin was determined to be approx. $0.9 \mathrm{meq} / \mathrm{g}$. The capacity of the Sarasep resin was found to be $0.2 \mathrm{meq} / \mathrm{g}$.

The apparatus for SPE consists of a $30 \mathrm{ml}$ glass reservoir connected by a small adapter to the SPE column itself. The SPE columns were obtained from Varian (Harbor City, CA, USA). Approximately $100 \mathrm{mg}$ of the resin was packed into the 55 by $6.5 \mathrm{~mm}$ I.D. columns. The Amberchrome resin was held in place above and below by $20 \mu \mathrm{m}$ polyethylene frits. The SRP resin was held in place by cutting a circular disk out of a Rainin Nylon-66 filter of $0.45 \mu \mathrm{M}$ pore size and sandwiching it between two of the polyethylene frits. The bed height of the amberchrome resin was about $15 \mathrm{~mm}$, while the SRP was about $10 \mathrm{~mm}$. The top of the reservoir was connected by a ground glass joint to an adjustable air 
pressure source. The applied air pressure was used to control the flow rate of solutions through the SPE column. The columns were connected to the laboratory made reservoir by an adapter (Alltech Assoc., Deerfield, IL USA). The concentrated phenol and neutral samples collected were analyzed using an HP 5880A gas chromatograph with a flame ionization detector, an HP 5880A Series level 4 integrator, and an HP 7637A automatic sample injector (Hewlett-Packard, Avondale, PA, USA). The capillary column used was a supelco (Bellefonte, PA, USA) SPB-1.

The carboxylic acids were analyzed by HPLC using an LKB 2150 HPLC pump, an Applied Biosystem 783A UV absorbance detector, and a shimadzu chromatopac C-R3A integrator. The separation column was a $5 \mathrm{~cm}$ column packed with $5 \mu \mathrm{m}$ sulfonated $(0.6 \mathrm{meq} / \mathrm{g})$ resin.

\section{Procedure for SPE}

Prior to initial use, the anion exchange columns were cleaned with acetonitrile and methanol. The columns were then treated with ca. $5 \mathrm{ml}$ of $\mathrm{NaOH}$ solution to ensure the resin was in the basic form.

The sample solutions were prepared by spiking a dilute methanol solution of several neutral and acidic compounds to the deionized water such that a final concentration of 
ca. 0.5 to $5 \mathrm{ppm}$ is attained. The $\mathrm{pH}$ of this solution was then raised to $\mathrm{ca}$. 11 with $\mathrm{NaOH}$. The sample was then put in the reservoir and passed through the resin at a flow rate of approximately $1 \mathrm{ml} / \mathrm{min}$. The column and reservoir were then rinsed with about $5 \mathrm{ml}$ of water.

The column was rinsed with $1 \mathrm{ml}$ of methylene chloride to elute the neutral fraction. This fraction was collected in a GC vial and spiked with $0.1 \mathrm{ml}$ of an quinoxaline or toluene internal standard solution. The vial was capped, mixed with an orbital stirrer, and analyzed by GC. A $1 \mu 1$ sample was injected with a split ratio of 1:40. Helium carrier gas was used at a flow rate of $1 \mathrm{ml} / \mathrm{min}$. The oven temperature was held initially at $50^{\circ} \mathrm{C}$ for two minutes, then ramped at $15^{\circ} \mathrm{C} / \mathrm{min}$ to a final temperature of $225^{\circ} \mathrm{C}$. A flame ionization detector was used. The acid fraction was then eluted with 1 $\mathrm{ml}$ of $0.1 \mathrm{M} \mathrm{HCl}$ in methanol. When the acid fraction contained phenols, the analysis was performed by GC as described for the neutral fraction. Recoveries were calculated by comparison of relative peak area to that of samples prepared and not subjected to SPE.

Samples containing carboxylic acids were eluted with 1 $\mathrm{mL}$ of $2 \mathrm{M} \mathrm{HCl}$ in methanol and analyzed by HPLC. A $5 \mu \mathrm{l}$ aliquot of the sample was injected onto a $5 \mathrm{~cm}$ laboratory 
prepared column of $1 \mathrm{meq} / \mathrm{g}$ sulfonated $5 \mu \mathrm{m}$ PS/DVB resin. Separations were performed using an aqueous eluant of $10 \%$ acetonitrile, 1 to $2 \%$ butanol, and $1 \mathrm{mM} \mathrm{H}_{2} \mathrm{SO}_{4}$. Quantitation was achieved by comparison of peak areas to known standards. 


\section{RESULT8 AND DI8CU88ION}

\section{Group separation of Acids and Neutrals}

In the previous section it was illustrated that partially sulfonated resins still have the ability to retain neutral molecules by hydrophobic interactions at unreacted sites on the resin particle. This ability can lead to adsorption in a dual mode, hydrophobic and electrostatic attractions thus allowing for group separations. In this section the same principles outlined in the base/neutral separations are used with anion exchange resins to perform acid/neutral separations. The $\mathrm{pH}$ of the sample solution is raised to a basic $\mathrm{pH}$ with $\mathrm{NaOH}$ to ensure that the acids are present as anions and thus retained at the ion exchange sites.

Figure 24 shows a gas chromatogram of the neutral fraction obtained when concentrated and eluted with $\mathrm{HCl}$ in methanol. The average recoveries $(n=5)$ of a large number of neutrals are shown in Table XIII. In most instances, the recoveries of the neutrals are good ( $90 \%)$.

Phenols are eluted with $1 \mathrm{ml} 0.1 \mathrm{M} \mathrm{HCl}$ in methanol. It has been shown (Chen and Fritz, unpublished results) that when relatively small acid concentrations are injected onto a GC column in small volumes $(\approx 1 \mu l)$, the column shows no 

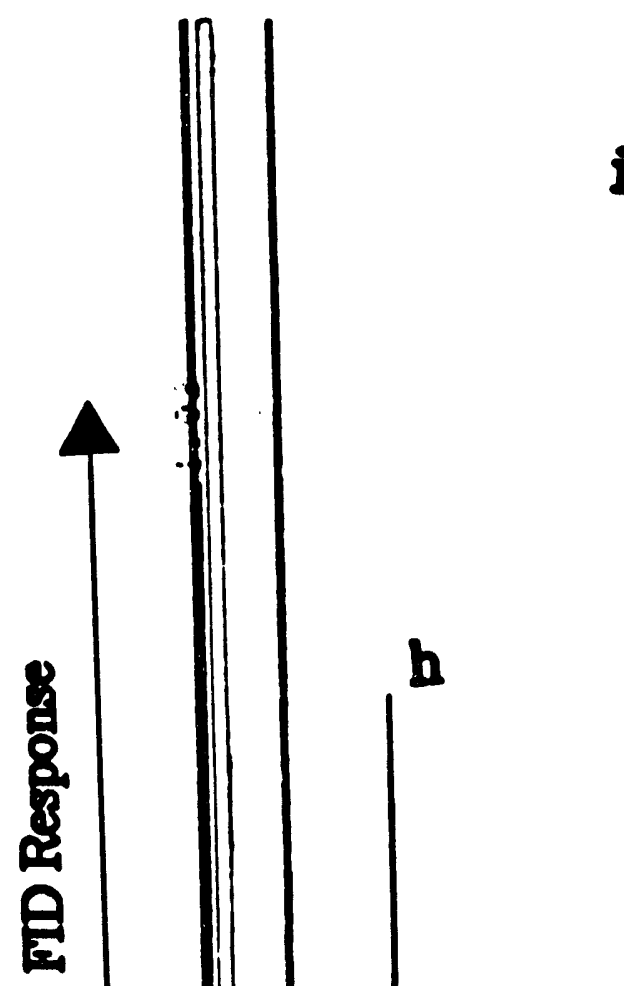

jk

h

1110

I

4

|

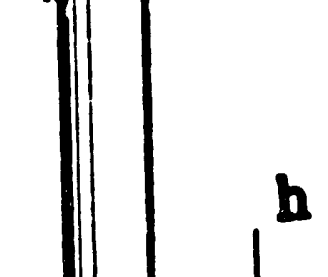

$+$

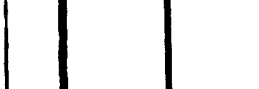


100

$\begin{array}{cc}\text { Table XIII. } & \text { Recoveries of neutrals }(0.5-5 \mathrm{ppm}) \text { from } 20 \mathrm{ml} \\ & \text { aqueous solutions using anion exchange resins. } \\ \text { Compound } & \& \text { Recovery }\end{array}$

Ethyl butyrate $\quad 101$

$\begin{array}{ll}\text { Chlorobenzene } & 87\end{array}$

$\begin{array}{ll}\text { Cyclohexanol } & 100\end{array}$

Bromohexane $\quad 90$

Salicyl aldehyde $\quad 99$

$\begin{array}{ll}\text { octanol } & 100\end{array}$

Nonyl aldehyde $\quad 100$

Triethyl orthopropionate $\quad 84$

$\begin{array}{ll}\text { Decanol } & 96\end{array}$

Benzyl alcohol 93

octyl aldehyde $\quad 92$

Nitrobenzene 96

Benzothiazole $\quad 103$

Toluene $\quad 93$

Anisole $\quad 96$ 
significant degradation over time. The small amount of acid present in these small injection volumes seems to be adsorbed onto the glass wool and silica contained in the glass liner of the injection port. This allows for the direct injection of the eluted acid fraction onto the GC column. Figure 25 shows the chromatugram obtained when the phenolic acid fraction is analyzed by GC. The resulting recoveries are shown in Table XIV.

When carboxylic acids were studied, analysis could not be performed by GC, due to the limited volatility of the underivatized sample. In this case the retained acids were quantified by ion exclusion chromatography on a laboratory prepared $5 \mathrm{~cm}$ sulfonated PS/DVB column. The carboxylic acids were eluted from the SPE column with $1 \mathrm{ml}$ of $1 \mathrm{M} \mathrm{HCl}$ in acetonitrile. This eluate was then injected directly onto the ion exclusion column for analysis. An example of the separation performed is shown in Figure 26 and recoveries given in Table $x V$.

\section{Group Separation of Acids and Neutrals in Non-polar Media}

Carboxylic acids can also be extracted from nonpolar solutions in a fashion similar to that illustrated with bases in section II. Carboxylic acids, being relatively involatile, cannot be injected onto a GC column without significant effects on the separation. This makes a method 


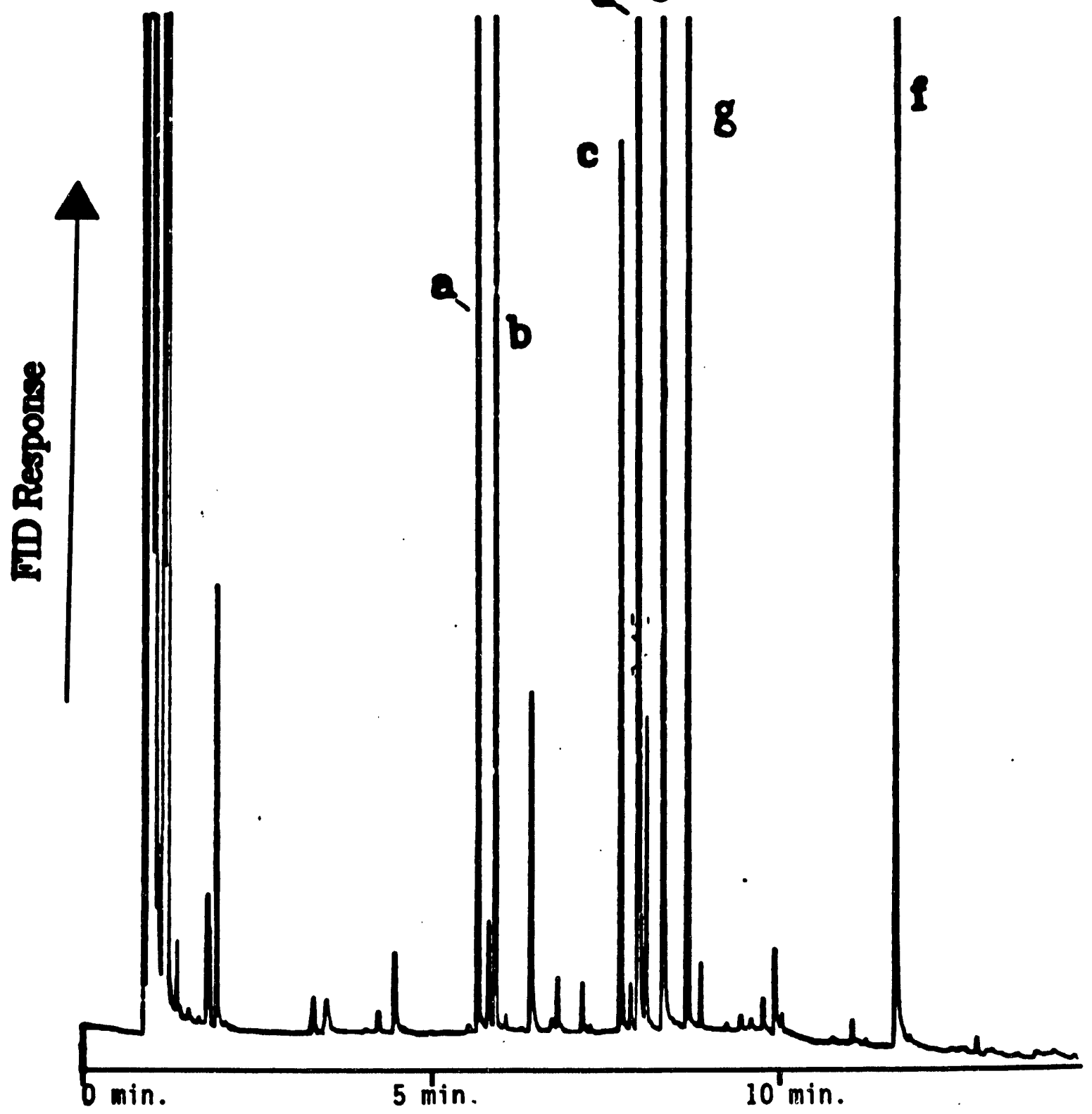

Figure 25. Gas chromatogram of acid (phenolic) fraction concentrated on anion exchange resin and eluted with $0.1 \mathrm{M} \mathrm{HCl}$ in methanol. ( $\mathrm{a}=$ phenol, $b=2$-chlorophenol, $c=4$-chlorophenol, $d=p-$

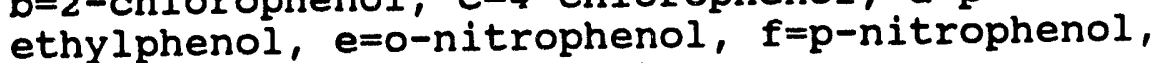
g=quinoxaline (int. std.\}) 
Table XIV. Percent recoveries of phenols (5 ppm) from $20 \mathrm{ml}$ aqueous solution using anion exchange resin.

Compound

\& Recovery

Phenol

2-Chlorophenol

4-Chlorophenol

o-Nitrophenol

m-Nitrophenol

p-Nitrophenol

p-Cresol

2,5-Dimethyl phenol

p-Isopropyl phenol

p-sec-Butyl phenol

p-Ethyl phenol
87

95

99

99

100

94

98

94

100

100

100 


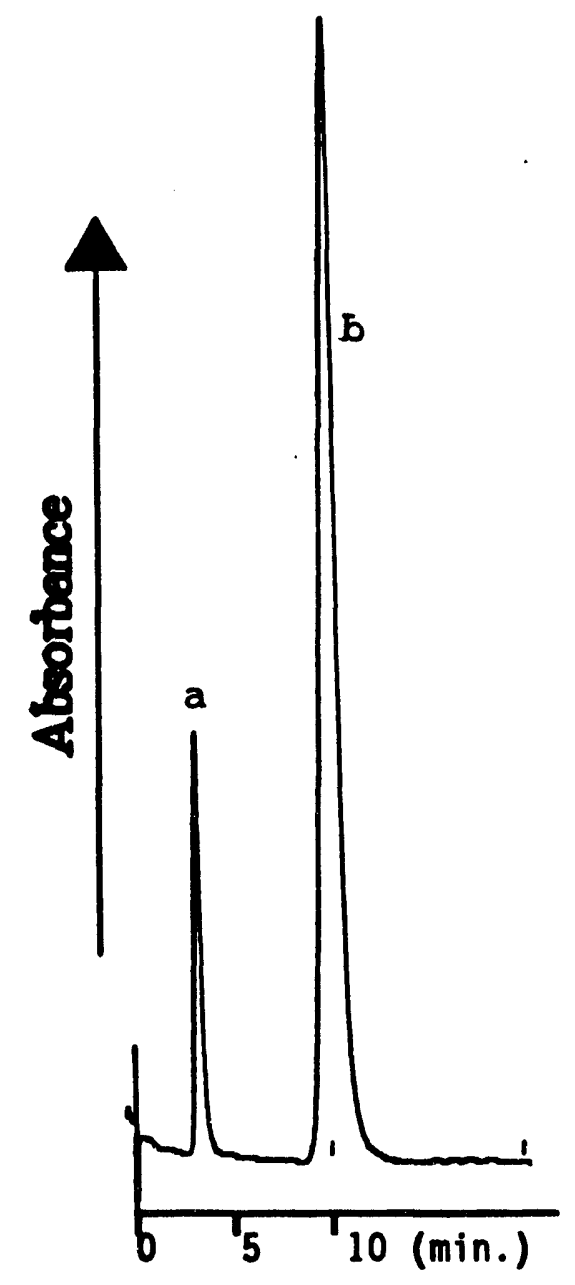

Figure 26. Liquid chromatogram of acidic (carboxylic acid) fraction concentrated on anion exchange resin and eluted with $0.1 \mathrm{M} \mathrm{HCl}$ in methanol. Acids shown are (a) hydroxy benzoic acid and (b) benzoic acid. 
Table XV. Percent recoveries of carboxylic acids (5-10ppm) phenols from aqueous solution using anion exchange resin.

Carboxylic acid

Hydroxy benzoic acid

Benzoic acid

p-Nitrobenzoic acid

$1,2,4$-Benzene tricarboxylic acid

Salicyl hydroxamic acid

Isonicotinic acid

2,4-Dihydroxy benzoic acid

Isophthalic acid

Phthalic acid
\& Recovery

99

96

99

105

102

95

99

100

100 
for their removal from solution to be analyzed by GC important. To $2 \mathrm{ml}$ of toluenesolution containing a mix of neutrals and carboxylic acids $0.1 \mathrm{ml}$ of $1 \mathrm{M}$ methyl amine was added in order to convert the acids to anions. This solution was then passed through an SPE column containing $\approx 10 \mathrm{mg}$ of the $5 \mu \mathrm{m}$ anion exchange resin to remove the acids. The neutral solution was then collected and analyzed by GC (Figure 27). The abstracted acids were then eluted from the column with $1 \mathrm{M} \mathrm{HCl}$ in methanol and analyzed by Ion Exclusion Chromatography as before. The recoveries of the neutrals and acids studied are shown in Tables XVI and XVII.

Though this type of separation may seem trivial, the presence of non-volatile carboxylic acids in samples to be analyzed by GC can be quite disastrous. Being semi-volatile, these acids become trapped at the head of a column, effectively ruining the column by causing problems with future separations, necessitating the removal of that portion of the column. 


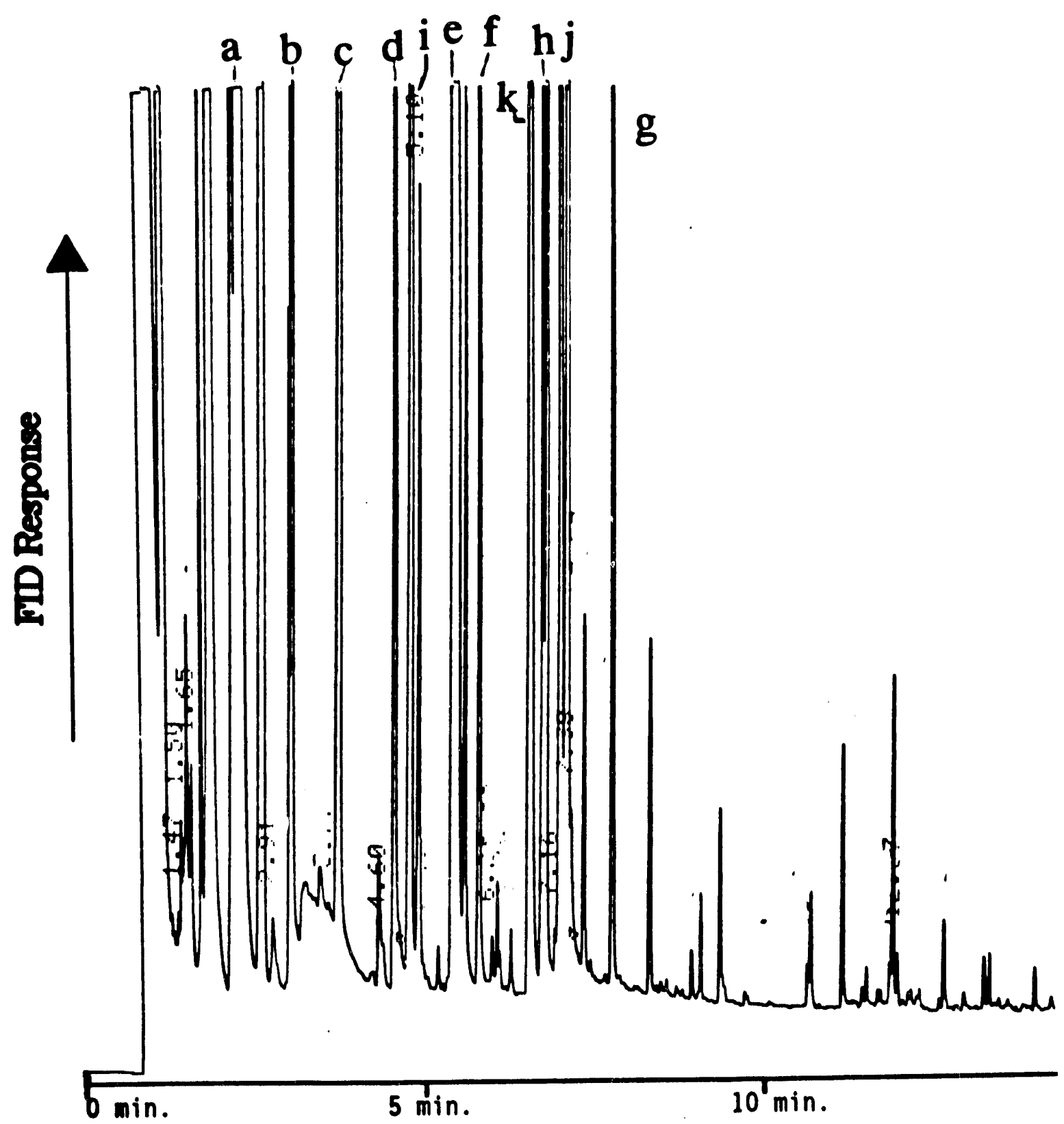

Figure 27. Gas chromatogram of neutral fraction after abstraction of carboxylic acids with anion exchange resin. \{a=ethyl butyrate, $b=$ chlorobenzene, $c=c y c l o h e x a n o l, d=b r o m o h e x a n e$, $e=s a l i c y l$ aldehyde, $f=o c t a n o l, g=n o n y l$ aldehyde, h=quinoxal ine (int. std.), $i=$ triethyl orthopropionate, j=decanol, and octyl aldehyde). 
Table XVI. Recoveries of neutrals (100 ppm) in $2 \mathrm{ml}$ acid/neutral group separation from toluene using anion exchange resin. Analysis by GC. $(n=5)$

\begin{tabular}{lcc} 
Neutral Compound & Recovery & Std. Deviation \\
\hline Ethyl butyrate & 103 & 5 \\
Chlorobenzene & 97 & 4 \\
Cyclohexanol & 99 & 3 \\
Bromohexane & 98 & 2 \\
Triethyl orthopropionate & 101 & 2 \\
Salicyl aldehyde & 94 & 10 \\
Octyl aldehyde & 107 & 5 \\
Decanol & 99 & 3 \\
Nonyl aldehyde & 98 & 3 \\
Octanol & 99 &
\end{tabular}


Table XVII. Recoveries of carboxylic acids (50 ppm) from $2 \mathrm{ml}$ acid/neutral mixture in toluene using anion exchange resin. Acids eluted with $\mathrm{HCl}$ in acetonitrile and analyzed by ion exclusion chromatography. $\quad(n=5)$

Carboxylic acid

\& Recovery Sta. Deviation

\begin{tabular}{lcc}
\hline Benzoic acid & 97 & 5 \\
2-Hydroxy benzoic acid & 98 & 1 \\
$1,2,4$-Benzene & 99 & 1 \\
tricarboxylic acid & 99 & 6 \\
$1,2,4,5$-Tetracarboxylic acid & 101 & 2 \\
$2,4-D i h y d r o x y$ benzoic acid & 98 & 5 \\
Phthalic acid & 98 & 1 \\
Isonicotinic acid & & 9 \\
\hline \hline
\end{tabular}




\section{CONCLUSION}

This type of concentration/group separation has many possible applications with both environmental and biological samples. Examples might include the cleanup and isolation of acidic drugs, phenolic pollutants, and humic acids. The group separation gives additional chemical information and provides another mode of separational ability. It allows complex samples containing compounds that must be analyzed by different methods to be separated prior to analysis.

The methods demonstrated were quick and precise, and the possibility of automation give them a bright future. Results were good for most compounds and reproducible. On-line separations, demonstrated in the last section for the separation of bases and neutrals, should be applicable in the separation of neutrals and acids as well. 


\section{GENERAL CONCLUSION}

Solid Phase Extraction (SPE) is fast becoming the preferred method for cleanup and concentration of liquid samples prior to chemical analysis. An increasing number of papers are published using these techniques for specific problems, and a large number of vendors are marketing disposable prepacked SPE tubes. Companies such as Bio-Rad (Richmond, CA), J. T. Baker (Phillipsburg, N.J.), and supelco, Inc. (Bellefonte, $P A$ ) have all published guides to applications of their SPE apparatus. As advances are made in the polymeric particles used, SPE may replace liquid/liquid extraction for routine analysis. The benefits of SPE by way of decreased solvent use, less labor involved, and higher extraction efficiencies give this technique promise.

one of the major concerns for the analytical chemist in today's world is the environment. As the list of chemical entities to be monitored increases, sample preparation techniques specific to certain classes of compounds become more important. The ability to selectively abstract and fractionate a class of pollutants can ease the demands on the analytical instrument. As the levels of these pollutants are often quite small with regards to other entities in the matrix, the ability to abstract and concentrate begins doubly important. often times the ill effects of pollutants are 
felt even at sub ppb levels. The old adage prevalent in industry with regard to the discharge of wastes, "dilution is the solution to pollution", may not always be true.

SPE is also finding uses in clinical laboratories. An increased awareness of the problems brought on by drugs in the workplace has created a need for drug testing in industry, and techniques ure needed to detect low levels of these drugs in biological fluids. A sample prep technique which can extract, concentrate, and fractionate drugs in urine or blood samples is needed. SPE is starting to fill this void.

The acetyl membrane demonstrated in this work shows promise in the environmental analysis of polar organics in aqueous solutions. Phenolic wastes have often been a thorn in the side of SPE. Being relatively hydrophilic, they give low recoveries when extracted with $\mathrm{si}-\mathrm{C}_{18}$ or underivatized polymeric resins. By chemically modifying the surface of the polymeric resin with a polar acetyl group, the resin was made more amenable to phenols present in water. This is supported by the fact that the acetyl resin showed good efficiency in the abstraction of phenols from deionized, tap, and river water samples.

By sulfonating a polymeric resin to produce a cation exchange resin, we were able to accomplish two things. The 
addition of the sulfonic acid group allows for the uptake of cations electrostatically, while allowing for the adsorption of neutrals at underivatized sites on the resin. The sulfonic acid groups on the resin increase the hydrophilicity of the resin, creating a more efficient interaction between solution and surface. The recoveries of both neutrals and protonated bases were quite good, and a complete group separation was performed. This type of separation is important in complicated sample mixtures and in the separation of classes of drugs. The ability to selectively abstract a species according to the $\mathrm{pk}_{\mathrm{b}}$ of the analyte can be a useful tool in the analysis of trace components in complex mixtures. The separation has been developed for both polar and apolar solutions, extending its application to all liquid samples. An on-line group separation has also been demonstrated. This type of work has a future in process analytical chemistry and the analytical laboratory. Any process that reduces the manual labor not only increases laboratory efficiency, but also cuts out analysis steps which may introduce errors in the final analysis.

The introduction of a quaternary ammonium group to the polymeric resin allows for the group separation of acid and neutrals. This type of separation is done in both clinical (acidic and neutral drugs) and environmental (phenols and 


\begin{abstract}
114
humic acids) analysis. The extraction of acids is especially important prior to analysis by gas chromatography, as the majority of acids are involatile and must be analyzed by alterior methods. Additionally, exposure to acids decreases the life of a GC column significantly. In the future, this type of abstraction could be done on-line to prevent the fouling of columns by tainted or acidic samples.
\end{abstract}




\section{REFERENCES}

(1) Liska, Krupcik, and Leclercq, J. High Resolution Chromatography, $1989,12,577$.

(2) J. R . Kaczvinsky, Jr., Koichi Saitoh, and James $s$. Fritz, Anal. Chem., 1983, 55, 1210.

(3) J. J. Richard, and Gregor A. Junk, Water Qual. Bull., 1981, Vol. 6, No. 2, 40-42, 54-55.

(4) M. Moors and D. L. Massart, Trends in Analytical Chemistry, 1990, Vol.9, No. $5,164$.

(5) G. A. Junk, J. J. Richard, M. D. Grieser, D. Witiak, J. L. Witiak, M. D. Arguello, R. Vick, H. J. Svec, J. S. Fritz and G. V. Calder, J. Chromatogr,, 1974, 99, 745.

(6) J.J. Sun and J.S. Fritz, J. Chromatogr., 1992, 590, 197.

(7) F. Nevejans and M. Verzele, J. Chromatogr., 1987, 406, 325 .

(8) R. Greyson and A. Patch, J. Chromatogr., $1982, \underline{242}, 349$.

(9) M. H. I. Baird Can. Journ. of Chem. Eng., 1991, 69, 1287 .

(10) D. Hagen, C. Markell, G. Schmitt, and D. Blevins, Anal. Chim. Acta, 1990, 236, 157.

(11) D. Barcelo, G. Durand, V. Bouvot, and M. Nielem, Env. Sci. and Tech., 1993, 27, 271. 
116

(12) S. Senseman, T. Lavy, J. Mattice, B. Myers, and B. Skulman, Env. Sci. Tech., 1993, 27, 516.

(13) M. Hennion, Trends in Anal. Chem., 1991, 10, 317.

(14) R. Majors and K. Fogelman, Amer. Lab., Feb., 1993, 40W.

(15) G. Junk, M. Avery, and J. J. Richard, Anal. Chem., 1988, 60,1347 .

(16) M. Chiba, etal. Anal. Chem., 1990, 1495.

(17) G. Junk and J. J. Richard, Anal. Chem., 1988, 60, 451.

(18) J. Sun and J. S. Fritz, J.Chromatogr., 1990, 522, 95.

(19) C. Markell and D. Hagen, LC/GC, 1991, 9, 332.

(20) Fed. Reg. 49, 58, (Oct. 26, 1984).

(21) V. Dixit and V. Dixit, Amer. Env. Lab., 1992, 4, 25.

(22) H. Svec and S. Colgrove, Anal. Chem., 1981, 53, 1737.

(23) M. Novotny and M. Lee, Journ. Chrom. Sci., 1974, 12, 606 .

(24) M. Fujimaki, K. Kim, and T. Kurata, Arric. Biol. Sci., $1974, \underline{38}, 45$.

(25) O. H. Wheeler, Chem. Rev, 1962, 62, 205.

(26) E. Durhan, M. Lukasewycz, and S. Baker, J. Chromatogr., $1993, \underline{629}, 67$.

(27) V. Lopez-Avila, J. Benedicto, and J. Milanes, EPA Report $600 / 4-89 / 049,124 \mathrm{pp}$.

(28) F. Ulberth and E. Ach.s, J. Chromatogr., 1990, 504, 202. 
(29) N. Theobald, Anal. Chim. Acta,, 1988, 204, 135.

(30) T. Aldridge and M. Oates, J. Forensic Sci., 1986, 31, 666 .

(31) A. Dicorcia and M. Marchetti, org. Micropollut. Aquat. Env., Proc. Eur. Symp., 1991, 37.

(32) N. Yamaga, etal., J. Chromatogr., 1987, 422, 25.

(33) J. Street, D. Trafford and H. Makin, J. Chromatogr., $1985, \underline{343}, 259$.

(34) S. Poole and C. Poole, Anal. Chim. Acta., 1990, 236, 3.

(35) D. Ewells, Pure App. Chem., 1988, 60, 1437.

(36) B. Thompson, etal., LC/GC, 1989, Z, 846.

(37) R. Patel, J. Benson and D. Hometchko, LC/GC, 1990, $\underline{8}, 152$.

(38) J. R. Kavczvinsky, K. Salton and J. S. Fritz, Anal. Chem., 1983, 55, 1210 .

(39) R. Parry, D. Gisch and G. Wachob, Journ. Liq. Chrom., $1987,10,2429$.

(40) J. Sun and J. S. Fritz., J.H. R. C. and C. C., 1991, 14,656 .

(41) R. Barron and J. S. Fritz, Reactive Polymers, 1983, 1,215 .

(42) J. Sun and J. S. Fritz., J.H.R. C. and C. C., 1992, 15, 605 . 
(43) R. Chang, C. Chriswell, and J. S. Fritz, Anal. Chem., $1975,47,1325$

(44) M. Mills and E. Thurman, Anal. Chem., 1992, 64, 1985.

(45) M. Chiba, C. D. Hall, C. Marvin and I. Brindle, Anal. Chem., $1990,62,1495$. 


\section{ACKNOWLEDGEMENTS}

This research was supported by a grant from the $3 \mathrm{M} \mathrm{CO}$. , St. Paul, MN, U.S.A. Additional help was given by Doug Gjerde of Sarasep, Inc, Santa Clara, CA. The work was performed at Ames Laboratory under contract no. W-7405-eng-82 with the U.S. Department of Energy. The United States Government has assigned the DOE Report number IS-T 1668 to this thesis.

The author would like to thank God above all, and the following people for their contributions to this work.

First thanks go to Dr. James S. Fritz. His guidance and direction over the years have been the driving force behind all this work. He is the person most responsible for teaching me how to think as an analytical chemist and perform research. In addition, he has been a good friend to whom I will always feel close.

I would also like to thank Dr. Houk, who I have always felt I could go to for advice, whether it be chemistry or otherwise. In addition, Dr. Johnson, Dr. Oulman, and Dr. Kraus have always made themselves available to me when I needed their assistance in any way.

Without my wife Andrea, these last couple of years would have been immeasurably more difficult, I cannot thank her enough for agreeing to live the life of a poor graduate student with me for the last several years. 
120

I would also like to thank my officemate of five years, roommate of two years, and friend for life John Morris. These last five years have been happy for me largely due to his companionship. Additionally, Nancy Benz, Dave MacGregor, Rich Roberts, Oden Warren, Alan Hayes and all group members, both past and present, have aided me in innumerable ways.

In conclusion, I wish to thank my parents and family, for encouraging my education over the years, and Dr. Max Pickerill, for introducing me to the fascinations and importance of chemistry. "Thank the chemist." 

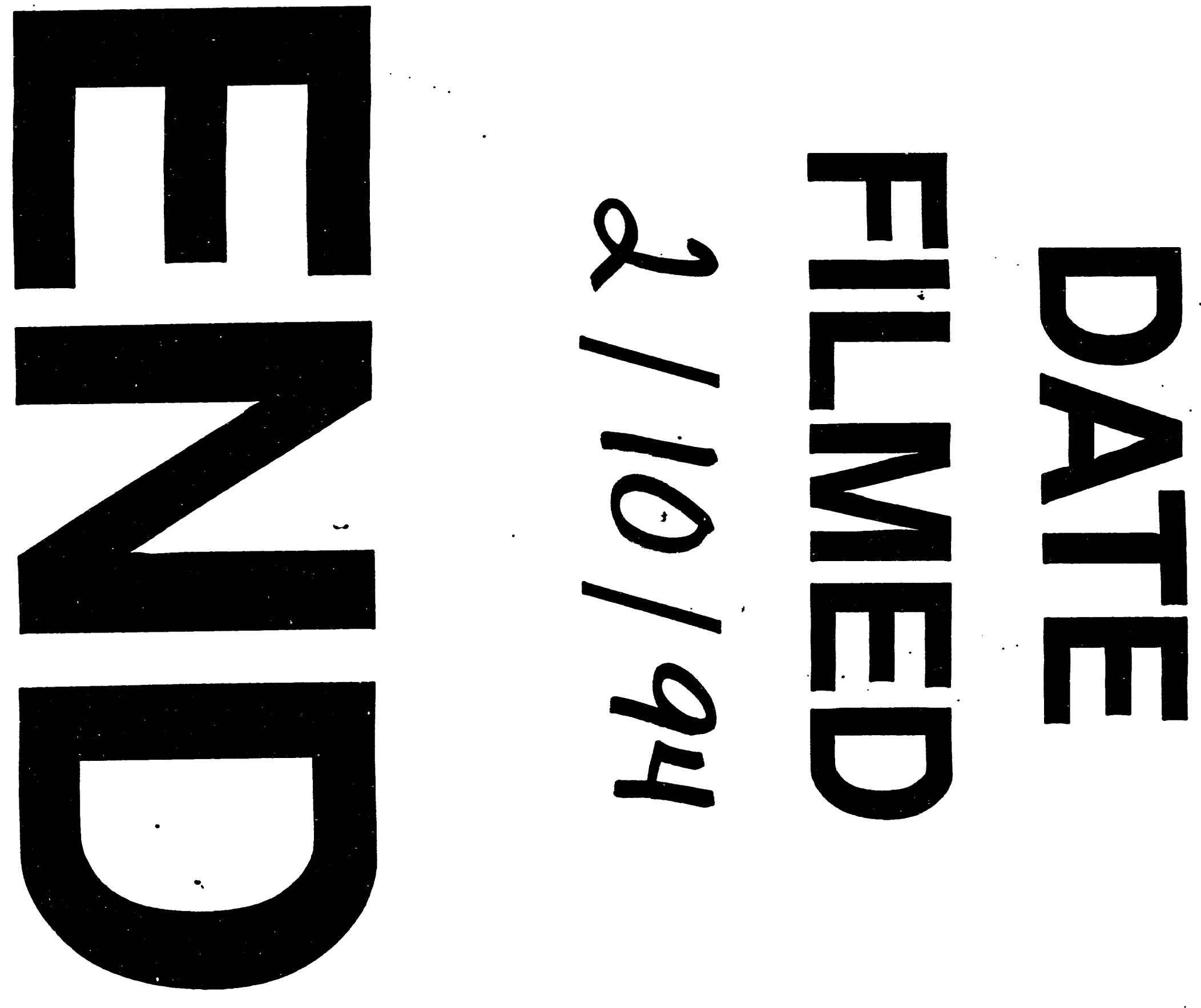

足 
I 